\title{
Hexacontaedro Pentagonal
}

\author{
Pentagonal Hexacontaedron
}

Recibido: mayo 11 de 2016 | Revisado: agosto 10 de 2016 | Aceptado: setiembre 15 de 2016

Erick J. Yarupaitan Mesías ${ }^{1}$

1 Universidad de San Martín de Porres,

Lima - Perú

erick4986@hotmail.com

\section{Resumen}

El objetivo de este estudio fue determinar las coordenadas de los 32 vértices de un hexacontaedro pentagonal para especificar la ubicación de los 32 transductores acústicos del Espejo Acústico con Inversión del Tiempo, mediante ecuaciones diferenciales e identidades trigonométricas, cuyas aplicaciones serán la recepción y transmisión invertida de señales acústicas, a través de un dispositivo electrónico de procesamiento para una media multi-objetivo de aplicación en el campo de la medicina, así como en comunicaciones submarinas y en sistemas de detección de aparición de fisuras en aeronaves. Como resultado del estudio se obtuvo las coordenadas esféricas de los 32 vértices equidistantes entre sí, y en relación con el centro del mismo.

Palabras clave: espejo acústico con inversión de tiempo, hexacontaedro pentagonal, transductores acústicos, ecuaciones diferenciales

\section{Abstract}

The aim of the study was to determine the coordinates of the 32 vertices of a pentagonal hexacontaedro to specify the location of the 32 acoustic transducers of the "Acoustic Mirror with Time Reversion" by differential equations and trigonometric identities, whose applications will be reverse reception and transmission of acoustic signals through an electronic processing device for a multi- objective media medical field, as well as submarine communications and systems to detect the appearance of fissures in aircrafts. As a result of the research, we obtained the spherical coordinates of the 32 vertexes, both equidistant from each other and in relation to the center of it.

Keywords: acoustic mirror with time reversion, pentagonal hexacontaedron, acoustic transducers, differential equations 


\section{Introducción}

La investigación Espejo Acústico con Inversión del Tiempo requirió, para su implementación, la adecuada ubicación de 32 transductores acústicos sobre la superficie de una esfera de un cierto radio.

Se observó que el dodecaedro regular cuenta con 20 caras y 12 vértices, por tanto, se optó por sobreponer, en sus 20 caras, sendas pirámides pentagonales cuyos vértices yacen sobre la misma superficie esférica en que se hallan circunscritos los 12 vértices iniciales, logrando así un hexacontaedro.

El hexacontaedro generado posee $60 \mathrm{ca}-$ ras y 32 vértices, pero no es regular ya que las caras triangulares de las pirámides pentagonales superpuestas no son equiláteras. $\mathrm{El}$ hexacontaedro producido luce similar a algo esférico, pero las coordenadas de sus 32 vértices corresponden a las ubicaciones requeridas para los transductores acústicos.

\section{Objetivo}

Determinar la ubicación de 32 transductores, perfectamente equidistantes del centro de una esfera y entre ellos.

\section{Desarrollo}

\section{Antecedentes}

El tetraedro, el hexaedro, el octaedro, el dodecaedro y el icosaedro, poliedros regulares de caras idénticas y con polígonos regulares del mismo tipo (Barriga \& Fayo, 2014), son los cinco sólidos ideales, estudiados por los antiguos griegos (López, 2007). Fue Pitágoras, quien llama al dodecaedro la "esfera de 12 pentágonos" (Mallo, 2015). Resulta oportuno y conveniente pasar revista a las características del dodecaedro, pues a partir de él, se creó el hexacontaedro pentagonal.
El sistema de coordenadas esféricas, al poseer simetría esférica, según Marsden y Tromba (1991) "simetría alrededor de un punto" (p. 51), se utiliza para determinar la posición espacial de un punto (Armenta, 2011) mediante una distancia y dos ángulos.

En consecuencia, un punto "P" queda representado por un conjunto de tres magnitudes: el radio "r", el ángulo polar " $\boldsymbol{\theta}$ " y el azimut “ $\varphi$ ".

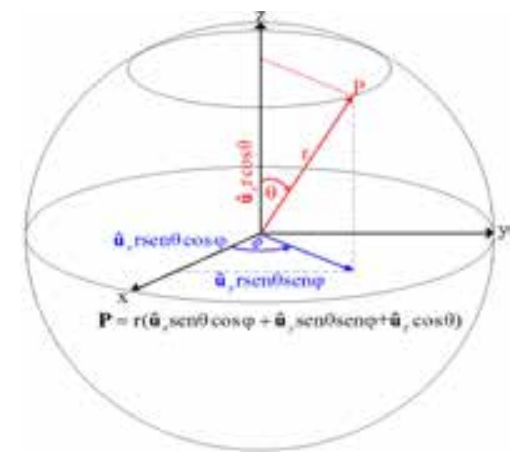

Figura 1. Representación de coordenadas esféricas

El radio vector $\mathbf{V}_{\mathrm{i}}$ del $\mathrm{i}$ - ésimo vértice viene dado por:

$$
\mathbf{V}_{\mathrm{i}}=\mathrm{r}\left[\hat{\mathbf{u}}_{x} \operatorname{sen}\left(\theta_{\mathrm{i}}\right) \cos \left(\varphi_{\mathrm{i}}\right)+\hat{\mathbf{u}}_{y} \operatorname{sen}\left(\theta_{\mathrm{i}}\right) \operatorname{sen}\left(\varphi_{\mathrm{i}}\right)+\hat{\mathbf{u}}_{z} \cos \left(\theta_{\mathrm{i}}\right)\right]
$$

En esta expresión "r" es el radio de la esfera; $\boldsymbol{\theta}_{\mathrm{i}}$ es el ángulo polar y $\boldsymbol{\varphi}_{\mathrm{i}}$, el acimut del vértice. Para el caso del dodecaedro, se supone cinco vértices, en el hemisferio norte, con un mismo ángulo polar $\boldsymbol{\theta}_{\mathrm{UP}} \mathrm{y}$ acimuts equi espaciados, cinco vértices en el hemisferio sur con un mismo ángulo polar $\pi-\boldsymbol{\theta}_{\mathrm{UP}} \mathrm{y}$ acimuts equi espaciados, cinco vértices en el hemisferio norte con un mismo ángulo polar $\boldsymbol{\theta}_{\mathrm{D}} \mathrm{y}$ acimuts equi espaciados y cinco

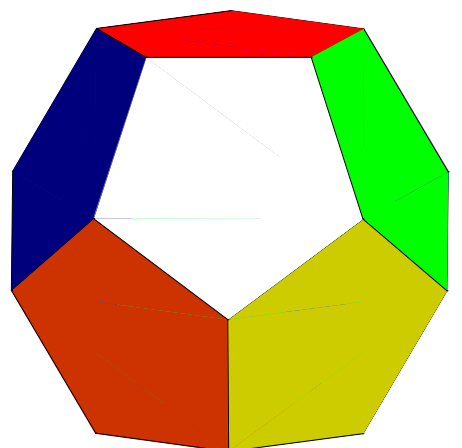

Figura 2. Representación pictórica del dodecaedro 
vértices en el hemisferio sur con un mismo ángulo polar $\pi-\theta_{\mathrm{D}} \mathrm{y}$ acimuts equi espaciados en perfecta simetría. Es decir:

$$
\begin{gathered}
\theta_{1}=\theta_{2}=\theta_{3}=\theta_{4}=\theta_{5}=\theta_{\mathrm{UP}} ; \\
\varphi_{1}=0, \varphi_{2}=2 \pi / 5, \varphi_{3}=4 \pi / 5, \varphi_{4}=6 \pi / 5, \varphi_{5}=8 \pi / 5 ; \\
\theta_{6}=\theta_{7}=\theta_{8}=\theta_{9}=\theta_{10}=\theta_{\mathrm{D}} ; \\
\varphi_{6}=0, \varphi_{7}=2 \pi / 5, \varphi_{8}=4 \pi / 5, \varphi_{9}=6 \pi / 5, \varphi_{10}=8 \pi / 5 ; \\
\theta_{11}=\theta_{12}=\theta_{13}=\theta_{14}=\theta_{15}=\pi-\theta_{\mathrm{D}} ; \\
\varphi_{11}=\pi / 5, \varphi_{12}=3 \pi / 5, \varphi_{13}=\pi, \varphi_{14}=7 \pi / 5, \varphi_{15}=9 \pi / 5 ; \\
\theta_{16}=\theta_{17}=\theta_{18}=\theta_{19}=\theta_{20} \equiv \pi-\theta_{\mathrm{UP}} ; \\
\varphi_{16}=\pi / 5, \varphi_{17}=3 \pi / 5, \varphi_{18}=\pi, \varphi_{19}=7 \pi / 5, \varphi_{20} \quad 9 \pi / 5 .
\end{gathered}
$$

Como las caras del dodecaedro son 12 pentágonos regulares idénticos, los vértices dados por $\mathbf{V}_{01}, \mathbf{V}_{02}, \mathbf{V}_{03}, \mathbf{V}_{04}$ y $\mathbf{V}_{05}$, en particular, definen un pentágono regular espaciados $2 \pi / 5$ con respecto al ángulo azimutal “ $\varphi$ ".

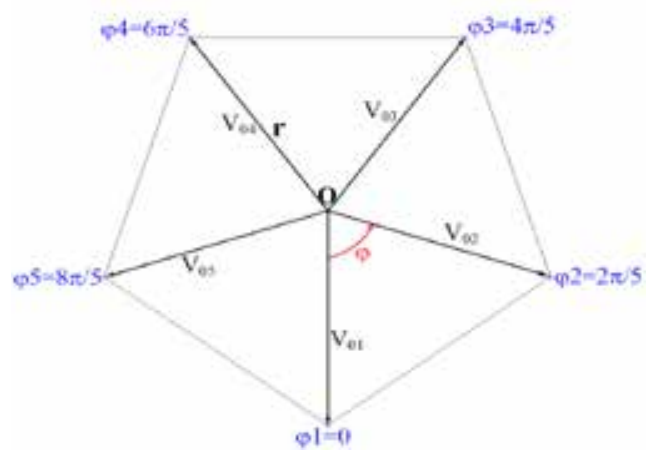

Figura 3. Vista superior de una cara del dodecaedro

Si se gira la cara superior del dodecaedro con respecto al ángulo polar, $\theta=\pi / 2$, se aprecia que los vectores $\mathrm{V}_{01}, \mathrm{~V}_{02}, \mathrm{~V}_{03}, \mathrm{~V}_{04}$ y $\mathrm{V}_{05}$ tienen como punto inicial el origen del dodecaedro y se encuentran en el ángulo polar $\theta=\theta_{\mathrm{UP}}$

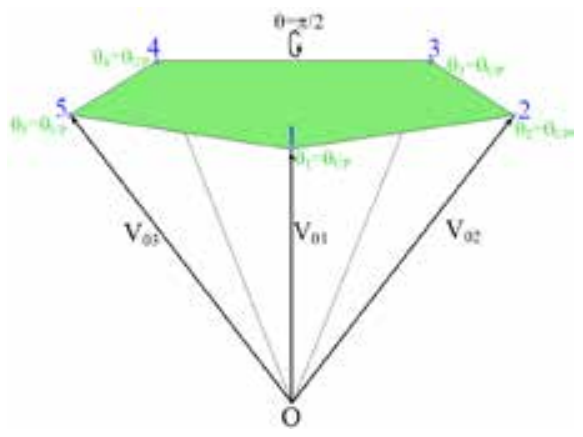

Figura 4. Cara superior del dodecaedro rotado $\theta=\pi / 2$
Si se incluyen las caras adyacentes a la cara superior del dodecaedro, se podrá visualizar que el vector $\mathrm{V}_{06}$ y el vector $\mathrm{V}_{01}$ se encuentran en el ángulo azimutal $\boldsymbol{\varphi}_{1}=\boldsymbol{\varphi}_{6}=0$.

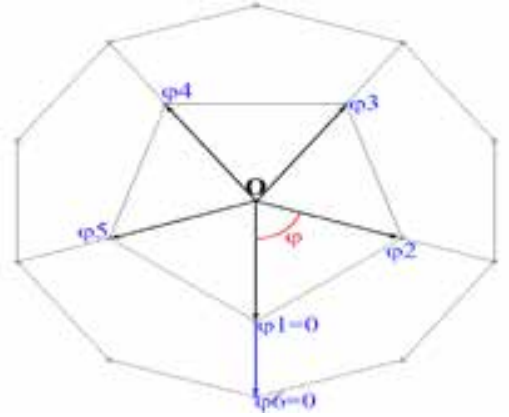

Figura 5. Cara superior del dodecaedro y caras adyacentes

Si se rota en $\theta=\pi / 2$, la cara superior del dodecaedro, incluidas las caras adyacentes, se aprecia que el vector $\mathrm{V}_{06}$ se encuentra en el ángulo polar $\theta_{6}=\theta_{D}$.

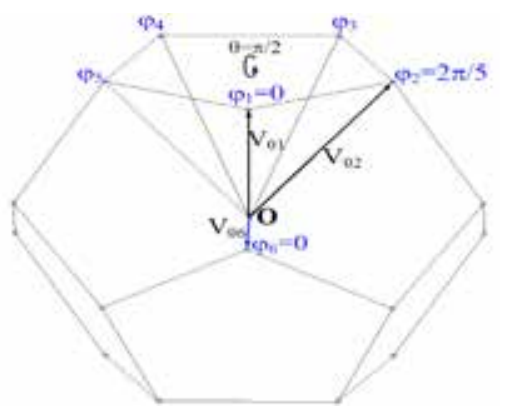

Figura 6. Cara superior del dodecaedro rotado $\mathrm{q}=\mathrm{p} / 2$ y caras adyacentes

Con el fin de visualizar correctamente los vectores $\mathrm{V}_{01} \mathrm{~V}_{02} \mathrm{yV}_{06}$ se rota alrededor del eje " $\mathrm{z}$ " un ángulo azimutal en $\mathrm{p} / 5$ según se muestra en la Figura 5.

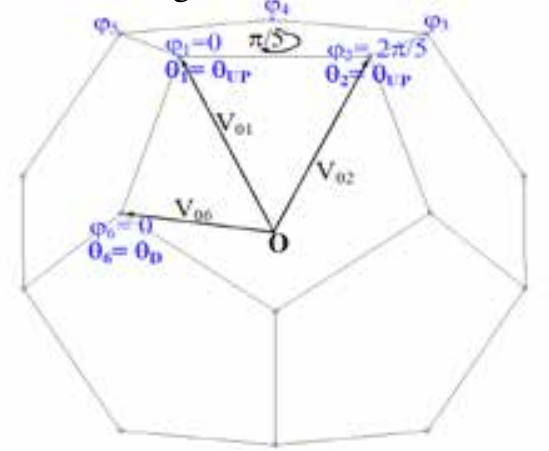

Figura 7. Caras adyacentes y superior del dodecaedro rotado con respecto al ángulo polar en $\theta=\frac{\pi}{2} \quad \mathrm{y}$ al ángulo azimutal en $\boldsymbol{\varphi}=\pi / 5$ 
Las ecuaciones correspondientes a los vértices $\mathbf{V}_{01}, \mathbf{V}_{02}, \mathbf{V}_{03}, \mathbf{V}_{04}, \mathbf{V}_{05} \mathbf{y} \mathbf{V}_{06}$ son:

$$
\begin{aligned}
& \mathbf{V}_{01}=\mathrm{r}\left[\hat{\mathbf{u}}_{x} \operatorname{sen}\left(\theta_{U P}\right) \cos (0)+\hat{\mathbf{u}}_{y} \operatorname{sen}\left(\theta_{U P}\right) \operatorname{sen}(0)+\hat{\mathbf{u}}_{z} \cos \left(\theta_{U P}\right)\right] \\
& \mathbf{V}_{01}=\mathrm{r}\left[\hat{\mathbf{u}}_{x} \operatorname{sen}\left(\theta_{U P}\right)+\hat{\mathbf{u}}_{z} \cos \left(\theta_{U P}\right)\right] \\
& \mathbf{V}_{02}=\mathrm{r}\left[\hat{\mathbf{u}}_{x} \operatorname{sen}\left(\theta_{U P}\right) \cos \left(\frac{2 \pi}{5}\right)+\hat{\mathbf{u}}_{y} \operatorname{sen}\left(\theta_{U P}\right) \operatorname{sen}\left(\frac{2 \pi}{5}\right)+\hat{\mathbf{u}}_{z} \cos \left(\theta_{U P}\right)\right] \\
& \mathbf{V}_{03}=\mathrm{r}\left[\hat{\mathbf{u}}_{x} \operatorname{sen}\left(\theta_{U P}\right) \cos \left(\frac{4 \pi}{5}\right)+\hat{\mathbf{u}}_{y} \operatorname{sen}\left(\theta_{U P}\right) \operatorname{sen}\left(\frac{4 \pi}{5}\right)+\hat{\mathbf{u}}_{z} \cos \left(\theta_{U P}\right)\right] \\
& \mathbf{V}_{04}=\mathrm{r}\left[\hat{\mathbf{u}}_{x} \operatorname{sen}\left(\theta_{U P}\right) \cos \left(\frac{6 \pi}{5}\right)+\hat{\mathbf{u}}_{y} \operatorname{sen}\left(\theta_{U P}\right) \operatorname{sen}\left(\frac{6 \pi}{5}\right)+\hat{\mathbf{u}}_{z} \cos \left(\theta_{U P}\right)\right] \\
& \mathbf{V}_{05}=\mathrm{r}\left[\hat{\mathbf{u}}_{x} \operatorname{sen}\left(\theta_{U P}\right) \cos \left(\frac{8 \pi}{5}\right)+\hat{\mathbf{u}}_{y} \operatorname{sen}\left(\theta_{U P}\right) \operatorname{sen}\left(\frac{8 \pi}{5}\right)+\hat{\mathbf{u}}_{z} \cos \left(\theta_{U P}\right)\right] \\
& \mathbf{V}_{06}=\mathrm{r}\left[\hat{\mathbf{u}}_{x} \operatorname{sen}\left(\theta_{D}\right) \cos (0)+\hat{\mathbf{u}}_{y} \operatorname{sen}\left(\theta_{D}\right) \operatorname{sen}(0)+\hat{\mathbf{u}}_{z} \cos \left(\theta_{D}\right)\right] \\
& \mathbf{V}_{06}=\mathrm{r}\left[\hat{\mathbf{u}}_{x} \operatorname{sen}\left(\theta_{D}\right)+\hat{\mathbf{u}}_{z} \cos \left(\theta_{D}\right)\right]
\end{aligned}
$$

Se obtiene el vector $\mathbf{V}_{02}-\mathbf{V}_{01}$ de (4) y (5):

$$
\begin{aligned}
& \mathbf{V}_{02}-\mathbf{V}_{01}=\mathrm{r}\left\{\hat{\mathbf{u}}_{x} \operatorname{sen}\left(\theta_{U P}\right)\left[\cos \left(\frac{2 \pi}{5}\right)-1\right]+\hat{\mathbf{u}}_{y} \operatorname{sen}\left(\theta_{U P}\right) \operatorname{sen}\left(\frac{2 \pi}{5}\right)\right\} \\
& \mathbf{V}_{02}-\mathbf{V}_{01}=\mathrm{r} \operatorname{sen}\left(\theta_{U P}\right)\left\{-\hat{\mathbf{u}}_{x}\left[1-\cos \left(\frac{2 \pi}{5}\right)\right]+\hat{\mathbf{u}}_{y} \operatorname{sen}\left(\frac{2 \pi}{5}\right)\right\}
\end{aligned}
$$

Se aplican las fórmulas E1 a: $1-\cos \left(\frac{2 \pi}{5}\right)$ y $\mathbf{E 2}$ a:

$\operatorname{sen}\left(\frac{2 \pi}{5}\right)$ para reducirlas a $\frac{\pi}{5}$ las que rinden:

$$
\begin{aligned}
& \mathbf{V}_{02}-\mathbf{V}_{01}=\mathrm{r} \operatorname{sen}\left(\theta_{U P}\right)\left[-\hat{\mathbf{u}}_{x} 2 \operatorname{sen}^{2}\left(\frac{\pi}{5}\right)+\hat{\mathbf{u}}_{y} 2 \operatorname{sen}\left(\frac{\pi}{5}\right) \cos \left(\frac{\pi}{5}\right)\right] \\
& \mathbf{V}_{02}-\mathbf{V}_{01}=2 \mathrm{r} \operatorname{sen}\left(\theta_{U P}\right) \operatorname{sen}\left(\frac{\pi}{5}\right)\left[-\hat{\mathbf{u}}_{x} \operatorname{sen}\left(\frac{\pi}{5}\right)+\hat{\mathbf{u}}_{y} \cos \left(\frac{\pi}{5}\right)\right] \therefore
\end{aligned}
$$

Dónde el módulo de $\mathbf{V}_{02}-\mathbf{V}_{01}$ es:

$$
\left|\mathbf{V}_{02}-\mathbf{V}_{01}\right|=2 \mathrm{r} \operatorname{sen}\left(\theta_{U P}\right) \operatorname{sen}\left(\frac{\pi}{5}\right) \therefore
$$

Y el vector unitario es:

$$
-\hat{\mathbf{u}}_{x} \operatorname{sen}\left(\frac{\pi}{5}\right)+\hat{\mathbf{u}}_{y} \cos \left(\frac{\pi}{5}\right)
$$

Luego se evalúa $\mathbf{V}_{06}-\mathbf{V}_{01}$ de (4) y (10):

$$
\mathbf{V}_{06}-\mathbf{V}_{01}=\mathrm{r}\left\{\hat{\mathbf{u}}_{x}\left[\operatorname{sen}\left(\theta_{D}\right)-\operatorname{sen}\left(\theta_{U P}\right)\right]+\hat{\mathbf{u}}_{z}\left[\cos \left(\theta_{D}\right)-\cos \left(\theta_{U P}\right)\right]\right\}
$$




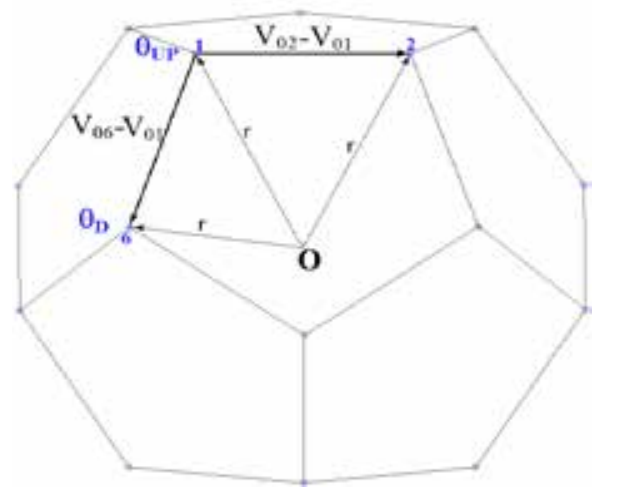

Figura 8. Vista de los lados formados por los vectores $\mathbf{V}_{06}-\mathbf{V}_{01}$ y $\mathbf{V}_{02}-\mathbf{V}_{01}$

Para relacionar $\theta_{D}$ y $\theta_{U P}$ se aplican las fórmulas (E7) que resulta de la combinación de la diferencia de senos y (E8) que resulta de la combinación de diferencia de cosenos que son:

$\operatorname{sen}(\alpha+\beta)-\operatorname{sen}(\alpha-\beta)=2 \cos \alpha \operatorname{sen} \beta$

$\cos (\alpha+\beta)-\cos (\alpha-\beta)=-2 \operatorname{sen} \alpha \operatorname{sen} \beta$

Se denomina a:

$\theta_{U P}=\alpha-\beta$

$\theta_{D}=\alpha+\beta$

Se trabajan las ecuaciones (18) y (19) que rinden:

$\theta_{U P}+\beta=\theta_{D}-\beta \Rightarrow 2 \beta=\theta_{D}-\theta_{U P}$

$\beta=\frac{\theta_{D}-\theta_{U P}}{2}$

Reemplazando (21) en (18):

$\theta_{U P}=\alpha-\frac{\theta_{D}-\theta_{U P}}{2} \Rightarrow 2 \theta_{U P}=2 \alpha+\theta_{U P}-\theta_{D}$

$\alpha=\frac{\theta_{D}+\theta_{U P}}{2}$

Reemplazando las fórmulas (18), (19), (22) y (23) en (E7):

$\operatorname{sen}\left(\theta_{D}\right)-\operatorname{sen}\left(\theta_{U P}\right)=2 \cos \left(\frac{\theta_{D}+\theta_{U P}}{2}\right) \operatorname{sen}\left(\frac{\theta_{D}-\theta_{U P}}{2}\right)$

Sustituyendo las fórmulas (20), (21), (24) y (25) en (E8):

$\cos \left(\theta_{D}\right)-\cos \left(\theta_{U P}\right)=-2 \operatorname{sen}\left(\frac{\theta_{D}+\theta_{U P}}{2}\right) \operatorname{sen}\left(\frac{\theta_{D}-\theta_{U P}}{2}\right)$

Conseguida la relación de $\theta_{D}$ y $\theta_{U P}$ se reemplaza (24) y

(25) en (17):

$\mathbf{V}_{06}-\mathbf{V}_{01}=\mathbf{r}\left\{\begin{array}{l}\hat{\mathbf{u}}_{x}\left[2 \cos \left(\frac{\theta_{D}+\theta_{U P}}{2}\right) \operatorname{sen}\left(\frac{\theta_{D}-\theta_{U P}}{2}\right)\right]+\ldots \\ \hat{\mathbf{u}}_{z}\left[-2 \operatorname{sen}\left(\frac{\theta_{D}+\theta_{U P}}{2}\right) \operatorname{sen}\left(\frac{\theta_{D}-\theta_{U P}}{2}\right)\right]\end{array}\right\}$ 


$$
\mathbf{V}_{06}-\mathbf{V}_{01}=2 \mathrm{r} \operatorname{sen}\left(\frac{\theta_{D}-\theta_{U P}}{2}\right)\left[\hat{\mathbf{u}}_{x} \cos \left(\frac{\theta_{D}+\theta_{U P}}{2}\right)-\hat{\mathbf{u}}_{z} \operatorname{sen}\left(\frac{\theta_{D}+\theta_{U P}}{2}\right)\right]
$$

Dónde el módulo de $\mathbf{V}_{06}-\mathbf{V}_{01}$ es:

$$
\left|\mathbf{V}_{06}-\mathbf{V}_{01}\right|=2 \mathrm{r} \operatorname{sen}\left(\frac{\theta_{D}-\theta_{U P}}{2}\right) \therefore
$$

Y el vector unitario es:

$$
-\hat{\mathbf{u}}_{x} \operatorname{sen}\left(\frac{\theta_{D}+\theta_{U P}}{2}\right)+\hat{\mathbf{u}}_{z} \operatorname{sen}\left(\frac{\theta_{D}+\theta_{U P}}{2}\right)
$$

Debido a que los lados del dodecaedro tienen la misma longitud, se cumple que los módulos son iguales, entonces se iguala (15) con (28):

$$
\begin{aligned}
& \left|\mathbf{V}_{02}-\mathbf{V}_{01}\right|=\left|\mathbf{V}_{06}-\mathbf{V}_{01}\right| \Rightarrow \\
& 2 r \operatorname{rsen}\left(\theta_{U P}\right) \operatorname{sen}\left(\frac{\pi}{5}\right)=2 \operatorname{rsen}\left(\frac{\theta_{D}-\theta_{U P}}{2}\right) \\
& \operatorname{sen}\left(\frac{\theta_{D}-\theta_{U P}}{2}\right)=\operatorname{sen}\left(\theta_{U P}\right) \operatorname{sen}\left(\frac{\pi}{5}\right) \therefore
\end{aligned}
$$

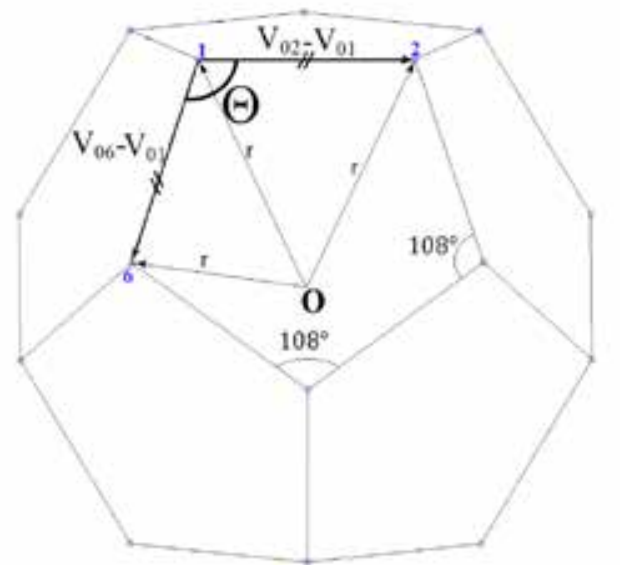

Figura 9 Módulo de $\mathbf{V}_{06}-\mathbf{V}_{01}$ igual al módulo de $\mathbf{V}_{02}-\mathbf{V}_{01}$

Según la fórmula (E10), para hallar el ángulo entre dos vectores:

$$
\frac{\mathrm{A} \cdot \mathrm{B}}{|\mathrm{A}| \cdot|\mathrm{B}|}=\cos \Theta
$$

Para este caso, se sabe que los vectores A y B son:

$$
\begin{aligned}
& A=V_{02}-V_{01} \\
& B=V_{06}-V_{01}
\end{aligned}
$$

En el Apéndice E, desde (E9) hasta (E12), en el cual se considera como dato que el ángulo $\Theta=\frac{3 \pi}{5}$ resultante de la intersección de los vectores A y B, se demuestra que el coseno de este ángulo $\Theta$ es:

$$
\cos \left(\frac{3 \pi}{5}\right)=-\cos \left(\frac{2 \pi}{5}\right) \therefore
$$

Reemplazando (14), (15), (27),(28) y (35) en (E10) 


$$
\begin{aligned}
& \frac{2 \mathrm{r} \operatorname{sen}\left(\theta_{\sigma P}\right) \operatorname{sen}\left(\frac{\pi}{5}\right)\left[-\hat{\mathbf{u}}_{x} \operatorname{sen}\left(\frac{\pi}{5}\right)+\hat{\mathbf{u}}_{y} \cos \left(\frac{\pi}{5}\right)\right]}{2 \mathrm{r} \operatorname{sen}\left(\theta_{O P}\right) \operatorname{sen}\left(\frac{\pi}{5}\right)} \ldots \\
& \frac{2 \operatorname{rsen}\left(\frac{\theta_{D}-\theta_{U P}}{2}\right)\left[\hat{\mathbf{u}}_{x} \cos \left(\frac{\theta_{D}+\theta_{U P}}{2}\right)-\hat{\mathbf{u}}_{z} \operatorname{sen}\left(\frac{\theta_{D}+\theta_{U P}}{2}\right)\right]}{2 \mathrm{r} \operatorname{sen}\left(\frac{\theta_{D}-\theta_{U P}}{2}\right)}=-\cos \left(\frac{2 \pi}{5}\right) \\
& {\left[-\hat{\mathbf{u}}_{x} \operatorname{sen}\left(\frac{\pi}{5}\right)+\hat{\mathbf{u}}_{y} \cos \left(\frac{\pi}{5}\right)\right] \cdots} \\
& {\left[\hat{\mathbf{u}}_{x} \cos \left(\frac{\theta_{D}+\theta_{U P}}{2}\right)-\hat{\mathbf{u}}_{z} \operatorname{sen}\left(\frac{\theta_{D}+\theta_{U P}}{2}\right)\right]=-\cos \left(\frac{2 \pi}{5}\right)}
\end{aligned}
$$

Aplicando la fórmula del producto escalar de vectores, adjunta en el Apéndice E, (E13) y (E14) se obtiene lo siguiente:

$$
\begin{aligned}
& -\operatorname{sen}\left(\frac{\pi}{5}\right) \cos \left(\frac{\theta_{D}+\theta_{U P}}{2}\right)=-\cos \left(\frac{2 \pi}{5}\right) \\
& \cos \left(\frac{\theta_{D}+\theta_{U P}}{2}\right)=\frac{\cos \left(\frac{2 \pi}{5}\right)}{\operatorname{sen}\left(\frac{\pi}{5}\right)} \\
& \theta_{D}+\theta_{U P}=2 \cos ^{-1}\left[\frac{\cos \left(\frac{2 \pi}{5}\right)}{\operatorname{sen}\left(\frac{\pi}{5}\right)}\right]
\end{aligned}
$$

Se presenta la forma de la ecuación (23) al primer término para usar la ecuación (32):

$$
\begin{aligned}
& \theta_{D}=2 \cos ^{-1}\left[\frac{\cos \left(\frac{2 \pi}{5}\right)}{\operatorname{sen}\left(\frac{\pi}{5}\right)}\right]-\theta_{U P} \\
& \frac{\theta_{D}-\theta_{U P}}{2}=\cos ^{-1}\left[\frac{\cos \left(\frac{2 \pi}{5}\right)}{\operatorname{sen}\left(\frac{\pi}{5}\right)}\right]-\theta_{U P}
\end{aligned}
$$

Reemplazando (42) en (32):

$$
\operatorname{sen}\left(\frac{\theta_{D}-\theta_{U P}}{2}\right)=\operatorname{sen}\left\{\cos ^{-1}\left[\frac{\cos \left(\frac{2 \pi}{5}\right)}{\operatorname{sen}\left(\frac{\pi}{5}\right)}\right]-\theta_{U P}\right\}=\operatorname{sen}\left(\theta_{U P}\right) \operatorname{sen}\left(\frac{\pi}{5}\right)
$$


Aplicando (E4):

$\operatorname{sen}\left(\theta_{U P}\right) \operatorname{sen}\left(\frac{\pi}{5}\right)=\operatorname{sen}\left\{\cos ^{-1}\left[\frac{\cos \left(\frac{2 \pi}{5}\right)}{\operatorname{sen}\left(\frac{\pi}{5}\right)}\right]\right\}$
$\cos \left(\theta_{U P}\right)-\frac{\cos \left(\frac{2 \pi}{5}\right)}{\operatorname{sen}\left(\frac{\pi}{5}\right)} \operatorname{sen}\left(\theta_{U P}\right)$
$\operatorname{sen}\left(\theta_{U P}\right)\left[\operatorname{sen}\left(\frac{\pi}{5}\right)+\frac{\cos \left(\frac{2 \pi}{5}\right)}{\left.\operatorname{sen}\left(\frac{\pi}{5}\right)\right]}=\operatorname{sen}\left\{\cos ^{-1}\left[\frac{\cos \left(\frac{2 \pi}{5}\right)}{\operatorname{sen}\left(\frac{\pi}{5}\right)}\right]\right\} \cos \left(\theta_{U P}\right)\right.$
$\operatorname{sen}\left(\theta_{U P}\right)\left[\frac{\operatorname{sen}^{2}\left(\frac{\pi}{5}\right)+\cos \left(\frac{2 \pi}{5}\right)}{\operatorname{sen}\left(\frac{\pi}{5}\right)}\right]=\operatorname{sen}\left\{\cos ^{-1}\left[\frac{\cos \left(\frac{2 \pi}{5}\right)}{\operatorname{sen}\left(\frac{\pi}{5}\right)}\right]\right\} \cos \left(\theta_{U P}\right)$

Aplicando (E15) en: $\cos \left(\frac{2 \pi}{5}\right)$ donde:

$u=\frac{\pi}{5}$

Se obtiene lo siguiente:

$$
\begin{aligned}
& \operatorname{sen}\left(\theta_{U P}\right)\left[\frac{\operatorname{sen}^{2}\left(\frac{\pi}{5}\right)+1-2 \operatorname{sen}^{2}\left(\frac{\pi}{5}\right)}{\operatorname{sen}\left(\frac{\pi}{5}\right)}\right]=\cdots \\
& \operatorname{sen}\left\{\cos ^{-1}\left[\frac{\cos \left(\frac{2 \pi}{5}\right)}{\operatorname{sen}\left(\frac{\pi}{5}\right)}\right]\right\} \cos \left(\theta_{U P}\right) \\
& \operatorname{sen}\left(\theta_{U P}\right)\left[\frac{1-\operatorname{sen}^{2}\left(\frac{\pi}{5}\right)}{\operatorname{sen}\left(\frac{\pi}{5}\right)}\right]=\operatorname{sen}\left\{\cos ^{-1}\left[\frac{\cos \left(\frac{2 \pi}{5}\right)}{\operatorname{sen}\left(\frac{\pi}{5}\right)}\right]\right\} \cos \left(\theta_{U P}\right) \\
& \tan \left(\theta_{U P}\right)=\frac{\operatorname{sen}\left(\frac{\pi}{5}\right)}{\cos ^{2}\left(\frac{\pi}{5}\right)} \operatorname{sen}\left\{\operatorname { c o s } ^ { - 1 } \left[\frac{\left.\cos \left(\frac{2 \pi}{5}\right)\right]}{\left.\left.\operatorname{sen}\left(\frac{\pi}{5}\right)\right]\right\}}\right.\right.
\end{aligned}
$$

Se denomina:

$$
\begin{aligned}
& \gamma=\cos ^{-1}\left[\frac{\cos \left(\frac{2 \pi}{5}\right)}{\operatorname{sen}\left(\frac{\pi}{5}\right)}\right] \\
& \tan \left(\theta_{U P}\right)=\frac{\operatorname{sen}\left(\frac{\pi}{5}\right)}{\cos ^{2}\left(\frac{\pi}{5}\right)} \operatorname{sen}(\gamma)
\end{aligned}
$$




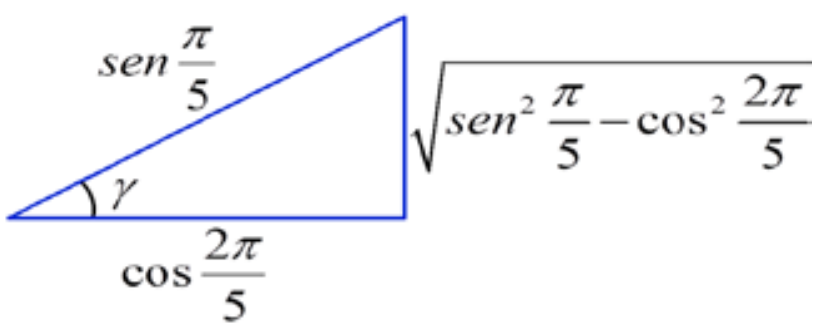

Figura 10 Representación del ángulo $\gamma$

En la Figura 10, se conoce que el cateto opuesto del ángulo $\gamma$ es:

$$
\begin{aligned}
& \sqrt{\operatorname{sen}^{2}\left(\frac{\pi}{5}\right)-\cos ^{2}\left(\frac{2 \pi}{5}\right)} \\
& \sqrt{\frac{1-\cos \left(\frac{2 \pi}{5}\right)}{2}-\cos ^{2}\left(\frac{2 \pi}{5}\right)} \\
& \frac{\sqrt{2}}{2} \sqrt{1-\cos \left(\frac{2 \pi}{5}\right)-2 \cos ^{2}\left(\frac{2 \pi}{5}\right)}
\end{aligned}
$$

Reemplazando (55) en (52):

$$
\begin{aligned}
& \tan \left(\theta_{U P}\right)=\frac{\operatorname{sen}\left(\frac{\pi}{5}\right)}{\cos ^{2}\left(\frac{\pi}{5}\right)} \times \frac{1}{\operatorname{sen}\left(\frac{\pi}{5}\right)} \frac{\sqrt{2}}{2} \sqrt{1-\cos \left(\frac{2 \pi}{5}\right)-2 \cos ^{2}\left(\frac{2 \pi}{5}\right)} \\
& \tan \left(\theta_{U P}\right)=\frac{\sqrt{2}}{2} \frac{1}{\cos ^{2}\left(\frac{\pi}{5}\right)} \sqrt{1-\cos \left(\frac{2 \pi}{5}\right)-2 \cos ^{2}\left(\frac{2 \pi}{5}\right)}
\end{aligned}
$$

En el apéndice A se demuestra que:

$$
\begin{aligned}
& \cos \left(\frac{2 \pi}{5}\right)=\frac{\sqrt{5}-1}{4} \\
& \cos ^{2}\left(\frac{2 \pi}{5}\right)=\frac{3-\sqrt{5}}{8} \\
& \cos \left(\frac{\pi}{5}\right)=\frac{\sqrt{5}+1}{4} \\
& \cos ^{2}\left(\frac{\pi}{5}\right)=\frac{3+\sqrt{5}}{8}
\end{aligned}
$$

Reemplazando (A1), (A2) y (A4) en (57):

$$
\begin{aligned}
& \tan \left(\theta_{U P}\right)=\frac{\sqrt{2}}{2} \frac{1}{\frac{3+\sqrt{5}}{8}} \sqrt{1-\frac{\sqrt{5}-1}{4}-\frac{\not 2(3-\sqrt{5})}{\not}} \\
& \tan \left(\theta_{U P}\right)=\frac{\sqrt{2}}{\not 2} \frac{1}{\frac{3+\sqrt{5}}{\not}} \sqrt{1-\frac{\sqrt{\not}}{4}+\frac{1}{4}-\frac{3}{4}+\frac{\sqrt{\not}}{4}}=\frac{4 \sqrt{2}}{3+\sqrt{5}} \frac{1}{\not \sqrt{2}} \\
& \tan \left(\theta_{U P}\right)=\frac{4}{3+\sqrt{5}}
\end{aligned}
$$

Racionalizando:

$$
\begin{aligned}
& \tan \left(\theta_{U P}\right)=\frac{4}{3+\sqrt{5}} \frac{3-\sqrt{5}}{3-\sqrt{5}}=\frac{12-4 \sqrt{5}}{9-5}=3-\sqrt{5} \\
& \tan \left(\theta_{U P}\right)=3-\sqrt{5} \\
& \theta_{U P}=\tan ^{-1}(3-\sqrt{5})
\end{aligned}
$$


Es más,

$$
\begin{aligned}
& \theta_{U P}=\tan ^{-1} \frac{2(3-\sqrt{5})}{2} \\
& \theta_{U P}=\tan ^{-1} 2 \mathscr{F}
\end{aligned}
$$

Es oportuno y conveniente señalar que $\mathrm{F}$ es un número de Fibonacci.

Reemplazando (63) en (41)

$$
\begin{aligned}
& \theta_{D}=2 \cos ^{-1}\left[\frac{\cos \left(\frac{2 \pi}{5}\right)}{\operatorname{sen}\left(\frac{\pi}{5}\right)}\right]-\theta_{U P} \\
& \theta_{D}=79.1877^{\circ} \\
& \pi-\theta_{D}=100.8123^{\circ}
\end{aligned}
$$

A partir de los valores, hallados en las ecuaciones (64), (65), (69) y (70), se obtienen los 20 vértices, en coordenadas esféricas del dodecaedro, desarrollados en el apéndice $\mathrm{F}$ y son los siguientes:

$$
\begin{aligned}
& \mathbf{V}_{01}=\mathrm{r}\left[\operatorname{sen}\left(\theta_{U P}\right), 0, \cos \left(\theta_{U P}\right)\right] \\
& \mathbf{V}_{02}=\mathrm{r}\left[\operatorname{sen}\left(\theta_{U P}\right) \cos \left(\frac{2 \pi}{5}\right), \operatorname{sen}\left(\theta_{U P}\right) \operatorname{sen}\left(\frac{2 \pi}{5}\right), \cos \left(\theta_{U P}\right)\right] \\
& \mathbf{V}_{03}=\mathrm{r}\left[-\operatorname{sen}\left(\theta_{U P}\right) \cos \left(\frac{\pi}{5}\right), \operatorname{sen}\left(\theta_{U P}\right) \operatorname{sen}\left(\frac{\pi}{5}\right), \cos \left(\theta_{U P}\right)\right] \\
& \mathbf{V}_{04}=\mathrm{r}\left[-\operatorname{sen}\left(\theta_{U P}\right) \cos \left(\frac{\pi}{5}\right),-\operatorname{sen}\left(\theta_{U P}\right) \operatorname{sen}\left(\frac{\pi}{5}\right), \cos \left(\theta_{U P}\right)\right] \\
& \mathbf{V}_{05}=\mathrm{r}\left[\operatorname{sen}\left(\theta_{U P}\right) \cos \left(\frac{2 \pi}{5}\right),-\operatorname{sen}\left(\theta_{U P}\right) \operatorname{sen}\left(\frac{2 \pi}{5}\right), \cos \left(\theta_{U P}\right)\right] \\
& \mathbf{V}_{06}=\mathrm{r}\left[\operatorname{sen}\left(\theta_{D}\right), 0, \cos \left(\theta_{D}\right)\right] \\
& \mathbf{V}_{07}=\mathrm{r}\left[\operatorname{sen}\left(\theta_{D}\right) \cos \left(\frac{2 \pi}{5}\right), \operatorname{sen}\left(\theta_{D}\right) \operatorname{sen}\left(\frac{2 \pi}{5}\right), \cos \left(\theta_{D}\right)\right] \\
& \mathbf{V}_{08}=\mathrm{r}\left[-\operatorname{sen}\left(\theta_{D}\right) \cos \left(\frac{\pi}{5}\right), \operatorname{sen}\left(\theta_{D}\right) \operatorname{sen}\left(\frac{\pi}{5}\right), \cos \left(\theta_{D}\right)\right] \\
& \mathbf{V}_{09}=\mathrm{r}\left[-\operatorname{sen}\left(\theta_{D}\right) \cos \left(\frac{\pi}{5}\right),-\operatorname{sen}\left(\theta_{D}\right) \operatorname{sen}\left(\frac{\pi}{5}\right), \cos \left(\theta_{D}\right)\right] \\
& \mathbf{V}_{10}=\mathrm{r}\left[\operatorname{sen}\left(\theta_{D}\right) \cos \left(\frac{2 \pi}{5}\right),-\operatorname{sen}\left(\theta_{D}\right) \operatorname{sen}\left(\frac{2 \pi}{5}\right), \cos \left(\theta_{D}\right)\right] \\
& \mathbf{V}_{11}=\mathrm{r}\left[\operatorname{sen}\left(\pi-\theta_{D}\right) \cos \left(\frac{\pi}{5}\right), \operatorname{sen}\left(\pi-\theta_{D}\right) \operatorname{sen}\left(\frac{\pi}{5}\right), \cos \left(\pi-\theta_{D}\right)\right] \\
& \mathbf{V}_{12}=r\left[-\operatorname{sen}\left(\pi-\theta_{D}\right) \cos \left(\frac{2 \pi}{5}\right), \operatorname{sen}\left(\pi-\theta_{D}\right) \operatorname{sen}\left(\frac{2 \pi}{5}\right), \cos \left(\pi-\theta_{D}\right)\right] \\
& \mathbf{V}_{13}=\mathrm{r}\left[-\operatorname{sen}\left(\pi-\theta_{D}\right), 0, \cos \left(\pi-\theta_{D}\right)\right]
\end{aligned}
$$




$$
\begin{aligned}
& \mathbf{V}_{14}=\mathrm{r}\left[-\operatorname{sen}\left(\pi-\theta_{D}\right) \cos \left(\frac{2 \pi}{5}\right),-\operatorname{sen}\left(\pi-\theta_{D}\right) \operatorname{sen}\left(\frac{2 \pi}{5}\right), \cos \left(\pi-\theta_{D}\right)\right] \\
& \mathbf{V}_{15}=\mathrm{r}\left[\operatorname{sen}\left(\pi-\theta_{D}\right) \cos \left(\frac{\pi}{5}\right),-\operatorname{sen}\left(\pi-\theta_{D}\right) \operatorname{sen}\left(\frac{\pi}{5}\right), \cos \left(\pi-\theta_{D}\right)\right] \\
& \mathbf{V}_{16}=\mathrm{r}\left[\operatorname{sen}\left(\pi-\theta_{U P}\right) \cos \left(\frac{\pi}{5}\right), \operatorname{sen}\left(\pi-\theta_{U P}\right) \operatorname{sen}\left(\frac{\pi}{5}\right), \cos \left(\pi-\theta_{U P}\right)\right] \\
& \mathbf{V}_{17}=\mathrm{r}\left[-\operatorname{sen}\left(\pi-\theta_{U P}\right) \cos \left(\frac{2 \pi}{5}\right), \operatorname{sen}\left(\pi-\theta_{U P}\right) \operatorname{sen}\left(\frac{2 \pi}{5}\right), \cos \left(\pi-\theta_{U P}\right)\right] \\
& \mathbf{V}_{18}=\mathrm{r}\left[-\operatorname{sen}\left(\pi-\theta_{U P}\right), 0, \cos \left(\pi-\theta_{U P}\right)\right] \\
& \mathbf{V}_{19}=\mathrm{r}\left[-\operatorname{sen}\left(\pi-\theta_{U P}\right) \cos \left(\frac{2 \pi}{5}\right),-\operatorname{sen}\left(\pi-\theta_{U P}\right) \operatorname{sen}\left(\frac{2 \pi}{5}\right), \cos \left(\pi-\theta_{U P}\right)\right](89) \\
& \mathbf{V}_{20}=\mathrm{r}\left[\operatorname{sen}\left(\pi-\theta_{U P}\right) \cos \left(\frac{\pi}{5}\right),-\operatorname{sen}\left(\pi-\theta_{U P}\right) \operatorname{sen}\left(\frac{\pi}{5}\right), \cos \left(\pi-\theta_{U P}\right)\right](90)
\end{aligned}
$$

En la Figura 12, se representa el dodecaedro descrito por las coordenadas cartesianas de la configuración (71) a (90) con una longitud arbitraria del radio de la esfera en que está circunscrito.

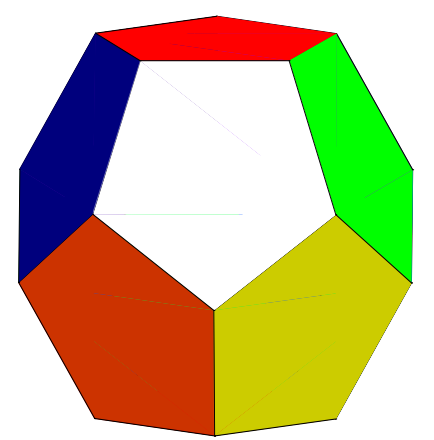

Figura 11. Representación pictórica del dodecaedro descrito por la configuración (71) a (90)

\section{Construcción del hexacontaedro pentagonal}

La configuración (2) establece que las coordenadas esféricas angulares del dodecaedro son las más convenientes para la construcción del hexacontaedro pentagonal, razón por la cual, se la transcribe con algunas variaciones menores en sus subíndices, a fin de facilitar el subsiguiente cálculo:

$$
\begin{gathered}
\theta_{1}=\theta_{2}=\theta_{3}=\theta_{4}=\theta_{5}=\theta_{\mathrm{UP}} ; \\
\varphi_{1}=0, \varphi_{2}=2 \pi / 5, \varphi_{3}=4 \pi / 5, \varphi_{4}=6 \pi / 5, \varphi_{5}=8 \pi / 5 ; \\
\theta_{6}=\theta_{7}=\theta_{8}=\theta_{9}=\theta_{10}=\theta_{\mathrm{D}} ; \\
\varphi_{6}=0, \varphi_{7}=2 \pi / 5, \varphi_{8}=4 \pi / 5, \varphi_{9}=6 \pi / 5, \varphi_{10}=8 \pi / 5 ; \\
\theta_{11}=\theta_{12}=\theta_{13}=\theta_{14}=\theta_{15} \equiv \pi-\theta_{\mathrm{D}} ; \\
\varphi_{11}=\pi / 5, \varphi_{12}=3 \pi / 5, \varphi_{13}=\pi, \varphi_{14}=7 \pi / 5, \varphi_{15}=9 \pi / 5 ; \\
\theta_{16}=\theta_{17}=\theta_{18}=\theta_{19}=\theta_{20} \equiv \pi-\theta_{\mathrm{UP}} ; \\
\varphi_{16}=\pi / 5, \varphi_{17}=3 \pi / 5, \varphi_{18}=\pi, \varphi_{19}=7 \pi / 5, \varphi_{20} 9 \pi / 5 .
\end{gathered}
$$


El cálculo de los radio-vectores de los centroides requeridos se efectuó, en forma detallada, en el Apéndice C, cuyo resultado se transcribe a través de un comentario.

El radio vector del centroide de la cara definida por los vértices $\mathbf{V}_{01}, \mathbf{V}_{02}, \mathbf{V}_{03}, \mathbf{V}_{04}$ y $\mathbf{V}_{05} \mathrm{y}$ su módulo se expresan en las siguientes ecuaciones (91) y (92):

$$
\begin{aligned}
& \mathbf{C}_{1}=\mathrm{r}\left[\hat{\mathbf{u}}_{z} \cos \left(\theta_{U P}\right)\right] \\
& \left|\mathbf{C}_{1}\right|=\mathrm{r}\left[\cos \left(\theta_{U P}\right)\right]
\end{aligned}
$$

El vector unitario del centroide es entonces:

$$
\hat{\mathbf{C}}_{1}=\frac{\not t\left[\hat{\mathbf{u}}_{z} \cos \left(\theta_{U P}\right)\right]}{\not t\left[\cos \left(\theta_{U P}\right)\right]}
$$

Como el vector unitario se presenta en coordenadas esféricas angulares, su componente z es el coseno del ángulo del vector, es decir:

$$
\cos \Theta_{1}=\frac{\cos \left(\theta_{U P}\right)}{\cos \left(\theta_{U P}\right)}=1
$$

A partir de la ecuación (94) se demuestra en el Apéndice C que:

$$
\Theta_{1}=0^{\circ}
$$

$$
\pi-\Theta_{1}=180^{\circ}
$$

De una manera totalmente análoga, se demuestra que el radio vector del centroide de la cara definida por los vértices $\mathbf{V}_{01}, \mathbf{V}_{02}, \mathbf{V}_{06}, \mathbf{V}_{07}$ y $\mathbf{V}_{11}$ y su módulo están dados por las ecuaciones (97) y (98):

$$
\begin{gathered}
\mathbf{C}_{2}=\frac{\mathrm{r}}{5}\left\{\begin{array}{l}
\hat{\mathbf{u}}_{x}\left[\operatorname{sen}\left(\theta_{U P}\right)\left(1+\cos \left(\frac{2 \pi}{5}\right)\right)+\operatorname{sen}\left(\theta_{D}\right)\left(1+\cos \left(\frac{2 \pi}{5}\right)+\cos \left(\frac{\pi}{5}\right)\right)\right] \ldots \\
+\hat{\mathbf{u}}_{y}\left[\operatorname{sen}\left(\theta_{U P}\right) \operatorname{sen}\left(\frac{2 \pi}{5}\right)+\operatorname{sen}\left(\theta_{D}\right)\left(\operatorname{sen}\left(\frac{2 \pi}{5}\right)+\operatorname{sen}\left(\frac{\pi}{5}\right)\right)\right]+\ldots \\
\hat{\mathbf{u}}_{z}\left[2 \cos \left(\theta_{U P}\right)+\cos \left(\theta_{D}\right)\right]
\end{array}\right\} \\
\left|\mathbf{C}_{2}\right|=\frac{\mathrm{r}}{5}\left\{\begin{array}{l}
{\left[\begin{array}{l}
\left.\operatorname{sen}\left(\theta_{U P}\right)\left(1+\cos \left(\frac{2 \pi}{5}\right)\right)+\operatorname{sen}\left(\theta_{D}\right)\left(1+\cos \left(\frac{2 \pi}{5}\right)+\cos \left(\frac{\pi}{5}\right)\right)\right]^{2} \\
{\left[\operatorname{sen}\left(\theta_{U P}\right) \operatorname{sen}\left(\frac{2 \pi}{5}\right)+\operatorname{sen}\left(\theta_{D}\right)\left(\operatorname{sen}\left(\frac{2 \pi}{5}\right)+\operatorname{sen}\left(\frac{\pi}{5}\right)\right)\right]^{2}+\ldots} \\
{\left[2 \cos \left(\theta_{U P}\right)+\cos \left(\theta_{D}\right)\right]^{2}}
\end{array}\right.}
\end{array}\right\}
\end{gathered}
$$

El vector unitario del centroide es entonces

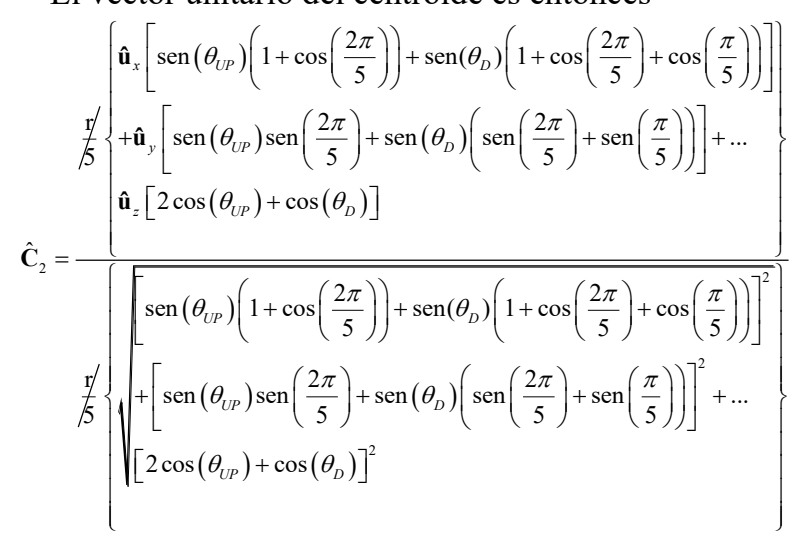


Como el vector unitario está expresado en coordenadas esféricas angulares su componente z es el coseno del ángulo del vector, es decir

$$
\cos \Theta_{2}=\frac{2 \cos \left(\theta_{U P}\right)+\cos \left(\theta_{D}\right)}{\left[\begin{array}{l}
\left.\operatorname{sen}\left(\theta_{U P}\right)\left(1+\cos \left(\frac{2 \pi}{5}\right)\right)+\operatorname{sen}\left(\theta_{D}\right)\left(1+\cos \left(\frac{2 \pi}{5}\right)+\cos \left(\frac{\pi}{5}\right)\right)\right]^{2} \\
+\left[\operatorname{sen}\left(\theta_{U P}\right) \operatorname{sen}\left(\frac{2 \pi}{5}\right)+\operatorname{sen}\left(\theta_{D}\right)\left(\operatorname{sen}\left(\frac{2 \pi}{5}\right)+\operatorname{sen}\left(\frac{\pi}{5}\right)\right)\right]^{2}+\ldots \\
{\left[2 \cos \left(\theta_{U P}\right)+\cos \left(\theta_{D}\right)\right]^{2}}
\end{array}\right.}
$$

A partir de la ecuación (100), se demuestra, en el Apéndice C que:

$$
\begin{aligned}
& \Theta_{2}=63.4349^{\circ} \\
& \pi-\Theta_{2}=116.5651^{\circ}
\end{aligned}
$$

La determinación de los respectivos acimuts se logra directamente por consideraciones de simetría, aunque puede obtenerse a partir de la razón de los componentes y sobre $\mathrm{x}$ de los radio-vectores $\mathbf{C}_{1}$ y $\mathbf{C}_{2}$.

\section{Resultados}

Las coordenadas esféricas angulares de los 32 vértices del hexacontaedro son las siguientes:

$$
\begin{gathered}
\theta_{1}=0^{\circ} ; \varphi_{1}=0^{\circ} ; \\
\theta_{2}=\theta_{3}=\theta_{4}=\theta_{5}=\theta_{6} \equiv 37^{\circ} ; \\
\varphi_{2}=36^{\circ}, \varphi_{3}=108^{\circ}, \varphi_{4}=180^{\circ}, \varphi_{5}=252^{\circ}, \varphi_{6}=324^{\circ} ; \\
\theta_{7}=\theta_{8}=\theta_{9}=\theta_{10}=\theta_{11} \equiv 63^{\circ} ; \\
\varphi_{7}=0^{\circ}, \varphi_{8}=72^{\circ}, \varphi_{9}=144^{\circ}, \varphi_{10}=216^{\circ}, \varphi_{11}=288^{\circ} ; \\
\theta_{12}=\theta_{13}=\theta_{14}=\theta_{15}=\theta_{16} \equiv 79^{\circ} ; \\
\varphi_{12}=36^{\circ}, \varphi_{13}=108^{\circ}, \varphi_{14}=180^{\circ}, \varphi_{15}=252^{\circ}, \varphi_{16}=324^{\circ} ; \\
\varphi_{17}=0^{\circ}, \varphi_{18}=72^{\circ}, \varphi_{19}=144^{\circ}, \varphi_{20}=216^{\circ}, \varphi_{21}=288^{\circ} ; \\
\varphi_{22}=36^{\circ}, \varphi_{23}=108^{\circ}, \varphi_{24}=180^{\circ}, \varphi_{25}=252^{\circ}, \varphi_{26}=324^{\circ} ; \\
\theta_{27}=\theta_{28}=\theta_{29}=\theta_{30}=\theta_{31} \equiv 143^{\circ} ; \\
\varphi_{27}=0^{\circ}, \varphi_{28}=72^{\circ}, \varphi_{29}=144^{\circ}, \varphi_{30}=216^{\circ}, \varphi_{31}=288^{\circ} ; \\
\theta_{32}=180^{\circ}
\end{gathered}
$$

En la Figura 12, se muestra el hexacontaedro, descrito en las coordenadas cartesianas de la configuración (103) con una longitud arbitraria del radio de la esfera en que se halla circunscrito. 


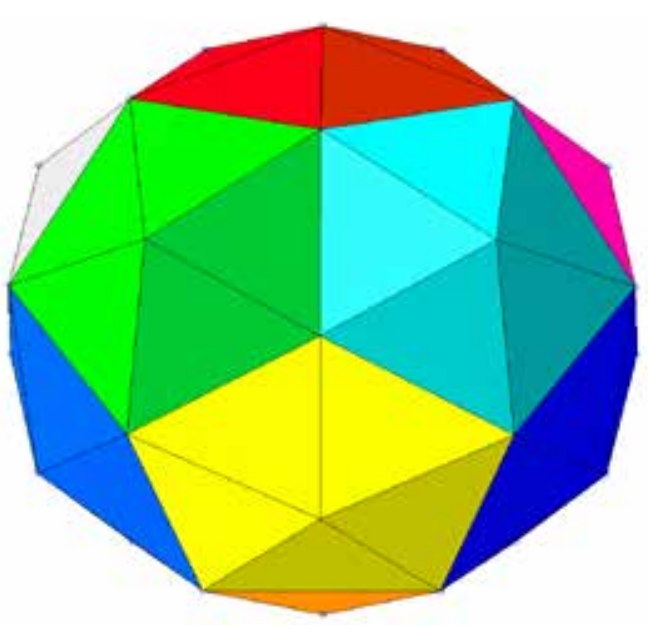

Figura 12. Hexacontaedro Pentagonal

\section{Conclusiones}

Las coordenadas de los 32 vértices del hexacontaedro pentagonal, dada su simetría, se utilizaron para ubicar a los 32 transductores acústicos del proyecto Espejo Acústico con Inversión del Tiempo, desarrollado por el
Instituto de Investigación de la Facultad de Ingeniería y Arquitectura de la Universidad de San Martín de Porres”. En la Figura 12, se muestra, pictóricamente, la posición de los 32 transductores del Espejo Acústico con Inversión del Tiempo.

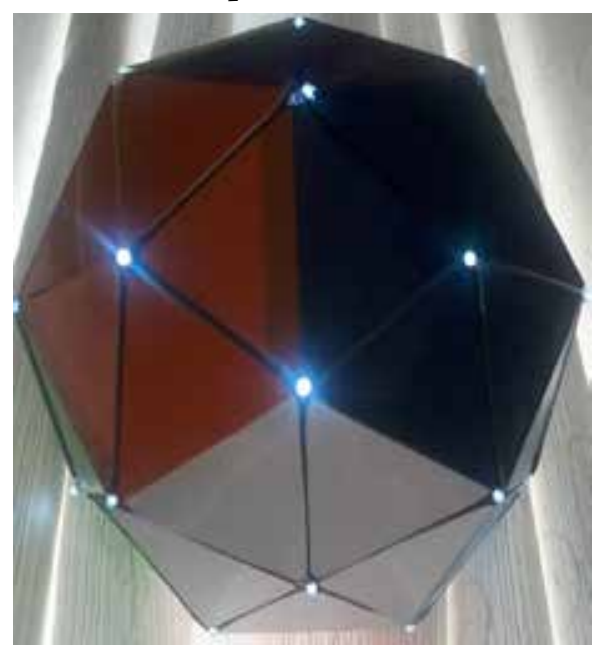

Figura 13. Posición de los 32 transductores representados en una led de una maqueta elaborada por el autor.

\section{Agradecimiento}

El autor expresa su agradecimiento a las autoridades de la Universidad de San Martín de Porres y de la Facultad de Ingeniería y Arquitectura por brindar las condiciones de trabajo requeridas para esta investigación.

Asimismo, nuestro reconocimiento póstumo al $\mathrm{PhD}$, Carlos Hugo Javier Calderón Chamochumbi, quien fuera investigador docente de esta casa de estudios, por su inapreciable apoyo y permanente asesoría científica en el campo matemático, por el uso liberal del apéndice A, sin el cual no se hubiese concretado esta investigación.

\section{Referencias}

Armenta, L. (2011). Diseño y construcción de una cabeza binaural (Tesis de pregrado, Instituto Politécnico Nacional Escuela Superior de Ingeniería Mecánica y Eléctrica, México). Recuperada de http://www.academia. edu/download/40497358/111.pdf

Barriga, E. \& Fayo, A. (2014). Conociendo el espacio a través de Cabri 3D. México: Devi-Kali.
López, S. (2007). Sólidos platónicos. Unión. Revista Iberoamericana de Educación Matemática, 12, 171-174. Recuperado de http://www.fisem.org/www/union/ revistas/2007/12/Union_012.pdf\#page $=157$

Mallo, M. (2015). Sistemas radiolarios: geometrias y arquitecturas derivadas (Tesis Doctoral, Universidad Politécnica de Madrid, España). Recuperado de: 
http://oa.upm.es/39863/1/MARIA_ MALLO_ZURDO_2a.pdf
Marsden, J. \& Tromba, A. (1991). Cálculo vectorial. Wilmington: AddisonWesley Iberoamericana.

\title{
ANEXOS
}

\author{
ANEXO A
}

(Propiedad del Profesor Carlos H. J. Calderón Chamochumbi, PhD )

$$
\begin{aligned}
& \operatorname{sen}\left(\frac{4 \pi}{5}+\frac{\pi}{5}\right)=\operatorname{sen}\left(\frac{4 \pi}{5}\right) \cos \left(\frac{\pi}{5}\right)+\cos \left(\frac{4 \pi}{5}\right) \operatorname{sen}\left(\frac{\pi}{5}\right)=0 \Rightarrow \\
& 2 \operatorname{sen}\left(\frac{2 \pi}{5}\right) \cos \left(\frac{2 \pi}{5}\right) \cos \left(\frac{\pi}{5}\right)+\operatorname{sen}\left(\frac{\pi}{5}\right)-2 \operatorname{sen}^{2}\left(\frac{2 \pi}{5}\right) \operatorname{sen}\left(\frac{\pi}{5}\right)=0 \Rightarrow \\
& 4 \operatorname{sen}\left(\frac{\pi}{5}\right)\left[1-2 \operatorname{sen}^{2}\left(\frac{\pi}{5}\right)\right] \cos ^{2}\left(\frac{\pi}{5}\right)+\operatorname{sen}\left(\frac{\pi}{5}\right)-8 \operatorname{sen}^{3}\left(\frac{\pi}{5}\right) \cos ^{2}\left(\frac{\pi}{5}\right)=0 \Rightarrow \\
& 4\left[1-2 \operatorname{sen}^{2}\left(\frac{\pi}{5}\right)\right] \cos ^{2}\left(\frac{\pi}{5}\right)+1-8 \operatorname{sen}^{2}\left(\frac{\pi}{5}\right) \cos ^{2}\left(\frac{\pi}{5}\right)=0 \Rightarrow \\
& 4 \cos ^{2}\left(\frac{\pi}{5}\right)-8 \operatorname{sen}^{2}\left(\frac{\pi}{5}\right) \cos ^{2}\left(\frac{\pi}{5}\right)+1-8 \operatorname{sen}^{2}\left(\frac{\pi}{5}\right) \cos ^{2}\left(\frac{\pi}{5}\right)=0 \Rightarrow \\
& 4-4 \operatorname{sen}^{2}\left(\frac{\pi}{5}\right)-8 \operatorname{sen}^{2}\left(\frac{\pi}{5}\right)+8 \operatorname{sen}^{4}\left(\frac{\pi}{5}\right)+1-8 \operatorname{sen}^{2}\left(\frac{\pi}{5}\right)+8 \operatorname{sen}^{4}\left(\frac{\pi}{5}\right)=0 \Rightarrow \\
& 5-20 \operatorname{sen}^{2}\left(\frac{\pi}{5}\right)+16 \operatorname{sen}^{4}\left(\frac{\pi}{5}\right)=0, \quad x \equiv \operatorname{sen}^{2}\left(\frac{\pi}{5}\right) \Rightarrow 16 x^{2}-20 x+5=0 \Rightarrow \\
& x=\frac{\sqrt{10 \pm\left(10^{2}-16 \times 5\right) \frac{1}{2}}}{16}=\frac{(5 \pm \sqrt{5})}{8} \Rightarrow \operatorname{sen}^{2}\left(\frac{\pi}{5}\right)=\frac{(5-\sqrt{5})}{8} \Rightarrow \\
& \operatorname{sen}\left(\frac{\pi}{5}\right)=\frac{\sqrt{2(5-\sqrt{5})}}{4} \Rightarrow \cos ^{2}\left(\frac{\pi}{5}\right)=1-\frac{(5-\sqrt{5})}{8}=\frac{3+\sqrt{5}}{8} \Rightarrow \\
& \cos ^{2}\left(\frac{\pi}{5}\right)=\frac{6+2 \sqrt{5}}{16}=\left[\frac{\sqrt{5}+1}{4}\right]^{2} \Rightarrow \cos \left(\frac{\pi}{5}\right)=\frac{\sqrt{5}+1}{4} \Rightarrow \\
& 2 \operatorname{sen}^{2}\left(\frac{\pi}{5}\right)=\frac{5-\sqrt{5}}{4} \Rightarrow \cos \left(\frac{2 \pi}{5}\right)=1-\frac{5-\sqrt{5}}{4} \Rightarrow \cos \left(\frac{2 \pi}{5}\right)=\frac{\sqrt{5}-1}{4} \Rightarrow \\
& \cos ^{2}\left(\frac{2 \pi}{5}\right)=\frac{6-2 \sqrt{5}}{16} \Rightarrow \operatorname{sen}^{2}\left(\frac{2 \pi}{5}\right)=1-\frac{6-2 \sqrt{5}}{16}=\frac{10+2 \sqrt{5}}{16} \Rightarrow \\
& \operatorname{sen}\left(\frac{2 \pi}{5}\right)=\frac{\sqrt{2(5+\sqrt{5})}}{4} \Rightarrow \operatorname{sen}\left(\frac{3 \pi}{5}\right)=\operatorname{sen}\left(\pi-\frac{2 \pi}{5}\right)=\operatorname{sen}\left(\frac{2 \pi}{5}\right) \Rightarrow
\end{aligned}
$$




$$
\begin{aligned}
& \operatorname{sen}\left(\frac{3 \pi}{5}\right)=\frac{\sqrt{2(5+\sqrt{5})}}{4}, \cos \left(\frac{3 \pi}{5}\right)=\cos \left(\pi-\frac{2 \pi}{5}\right)=-\cos \left(\frac{2 \pi}{5}\right) \Rightarrow \\
& \cos \left(\frac{3 \pi}{5}\right)=-\frac{\sqrt{5}-1}{4}, \operatorname{sen}\left(\frac{4 \pi}{5}\right)=\operatorname{sen}\left(\pi-\frac{\pi}{5}\right)=\operatorname{sen}\left(\frac{\pi}{5}\right) \Rightarrow \operatorname{sen}\left(\frac{4 \pi}{5}\right)=\frac{\sqrt{2(5-\sqrt{5})}}{4} \\
& \cos \left(\frac{4 \pi}{5}\right)=\cos \left(\pi-\frac{\pi}{5}\right)=-\cos \left(\frac{\pi}{5}\right) \Rightarrow \cos \left(\frac{4 \pi}{5}\right)=-\frac{\sqrt{5}+1}{4}, \\
& \operatorname{sen}\left(\frac{6 \pi}{5}\right)=\operatorname{sen}\left(2 \pi-\frac{4 \pi}{5}\right)=-\operatorname{sen}\left(\frac{4 \pi}{5}\right) \Rightarrow \operatorname{sen}\left(\frac{6 \pi}{5}\right)=-\frac{\sqrt{2(5-\sqrt{5})}}{4}, \\
& \cos \left(\frac{6 \pi}{5}\right)=\cos \left(2 \pi-\frac{4 \pi}{5}\right)=\cos \left(\frac{4 \pi}{5}\right) \Rightarrow \cos \left(\frac{6 \pi}{5}\right)=-\frac{\sqrt{5}+1}{4} \\
& \cos \left(\frac{7 \pi}{5}\right)=\cos \left(2 \pi-\frac{3 \pi}{5}\right)=\cos \left(\frac{3 \pi}{5}\right) \Rightarrow \cos \left(\frac{7 \pi}{5}\right)=-\frac{\sqrt{5}-1}{4}, \\
& \operatorname{sen}\left(\frac{7 \pi}{5}\right)=\operatorname{sen}\left(2 \pi-\frac{3 \pi}{5}\right)=-\operatorname{sen}\left(\frac{3 \pi}{5}\right) \Rightarrow \operatorname{sen}\left(\frac{7 \pi}{5}\right)=-\frac{\sqrt{2(5+\sqrt{5})}}{4}, \\
& \cos \left(\frac{8 \pi}{5}\right)=\cos \left(2 \pi-\frac{2 \pi}{5}\right)=\cos \left(\frac{2 \pi}{5}\right) \Rightarrow \cos \left(\frac{8 \pi}{5}\right)=\frac{\sqrt{5}-1}{4}, \\
& \operatorname{sen}\left(\frac{8 \pi}{5}\right)=\operatorname{sen}\left(2 \pi-\frac{2 \pi}{5}\right)=-\operatorname{sen}\left(\frac{2 \pi}{5}\right) \Rightarrow \operatorname{sen}\left(\frac{8 \pi}{5}\right)=-\frac{\sqrt{2(5+\sqrt{5})}}{4}, \\
& \cos \left(\frac{9 \pi}{5}\right)=\cos \left(2 \pi-\frac{\pi}{5}\right)=\cos \left(\frac{\pi}{5}\right) \Rightarrow \cos \left(\frac{9 \pi}{5}\right)=\frac{\sqrt{5}+1}{4}, \\
& \operatorname{sen}\left(\frac{9 \pi}{5}\right)=\operatorname{sen}\left(2 \pi-\frac{\pi}{5}\right)=-\operatorname{sen}\left(\frac{\pi}{5}\right) \Rightarrow \operatorname{sen}\left(\frac{9 \pi}{5}\right)=-\frac{\sqrt{2(5-\sqrt{5})}}{4}, \\
& \cos \left(\frac{\pi}{5}\right)=\frac{\sqrt{5}+1}{4}, \quad \operatorname{sen}\left(\frac{\pi}{5}\right)=\frac{\sqrt{2(5-\sqrt{5})}}{4} \quad * \\
& \cos \left(\frac{2 \pi}{5}\right)=\frac{\sqrt{5}-1}{4}, \quad \operatorname{sen}\left(\frac{2 \pi}{5}\right)=\frac{\sqrt{2(5+\sqrt{5})}}{4} \quad * \\
& \cos \left(\frac{3 \pi}{5}\right)=-\cos \left(\frac{2 \pi}{5}\right), \quad \operatorname{sen}\left(\frac{3 \pi}{5}\right)=\operatorname{sen}\left(\frac{2 \pi}{5}\right) \quad * \\
& \cos \left(\frac{4 \pi}{5}\right)=-\cos \left(\frac{\pi}{5}\right), \quad \operatorname{sen}\left(\frac{4 \pi}{5}\right)=\operatorname{sen}\left(\frac{\pi}{5}\right) \quad * \\
& \cos \left(\frac{6 \pi}{5}\right)=-\cos \left(\frac{\pi}{5}\right), \quad \operatorname{sen}\left(\frac{6 \pi}{5}\right)=-\operatorname{sen}\left(\frac{\pi}{5}\right) \quad * \\
& \cos \left(\frac{7 \pi}{5}\right)=-\cos \left(\frac{2 \pi}{5}\right), \quad \operatorname{sen}\left(\frac{7 \pi}{5}\right)=-\operatorname{sen}\left(\frac{2 \pi}{5}\right) \quad *
\end{aligned}
$$




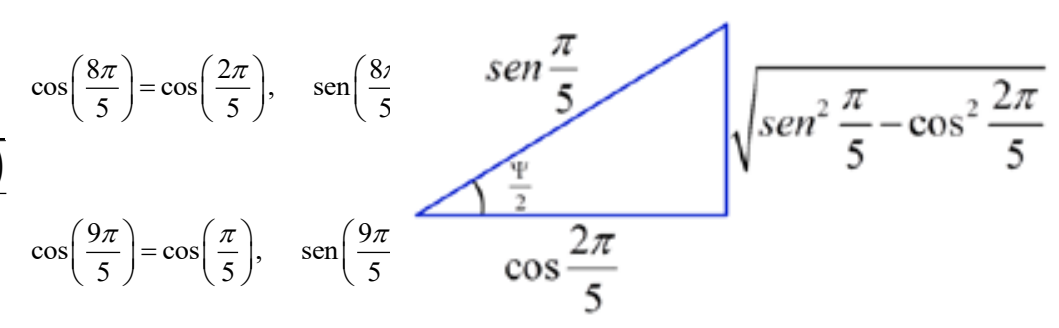

APÉNDICE B

De la ecuación (62) se desprende que:

$\tan \left(\theta_{U P}\right)=3-\sqrt{5}$

$\tan ^{2}\left(\theta_{U P}\right)=14-6 \sqrt{5}$

Figura 14 Representación del ángulo $\frac{\Psi}{2}$

$1+\tan ^{2}\left(\theta_{U P}\right)=15-6 \sqrt{5}$

Entonces:

$\theta_{D}=\Psi-\theta_{U P}$

$\cos \left(\theta_{D}\right)=\cos (\Psi) \cos \left(\theta_{U P}\right)+\operatorname{sen}(\Psi) \operatorname{sen}\left(\theta_{U P}\right)$

$\sec ^{2}\left(\theta_{U P}\right)=15-6 \sqrt{5}$

$\cos ^{2}\left(\theta_{U P}\right)=\frac{1}{15-6 \sqrt{5}}$

$\cos ^{2}\left(\theta_{U P}\right)=\frac{1}{3(5-2 \sqrt{5})} \times \frac{(5+2 \sqrt{5})}{(5+2 \sqrt{5})}$

Aplicando (E17) a $\left(\frac{\Psi}{2}\right)$ se tiene:

$\cos \left(\frac{\Psi}{2}\right)= \pm \sqrt{\frac{1+\cos (\Psi)}{2}}$

$\cos (\Psi)=2 \cos ^{2}\left(\frac{\Psi}{2}\right)-1$

$\cos ^{2}\left(\theta_{U P}\right)=\frac{5+2 \sqrt{5}}{15}$

$\cos (\Psi)=2\left[\frac{\cos \left(\frac{2 \pi}{5}\right)}{\operatorname{sen}\left(\frac{\pi}{5}\right)}\right]^{2}-1$

$\operatorname{sen}^{2}\left(\theta_{U P}\right)=1-\frac{5+2 \sqrt{5}}{15}$

$\operatorname{sen}^{2}\left(\theta_{U P}\right)=\frac{10-2 \sqrt{5}}{15}$

$\operatorname{sen}\left(\theta_{U P}\right)=\sqrt{\frac{10-2 \sqrt{5}}{15}}$

$\cos (\Psi)=2\left[\frac{\frac{\sqrt{5}-1}{A}}{\frac{\sqrt{2(5-\sqrt{5})}}{A}}\right]^{2}-1$

APÉNDICE C

$\cos (\Psi)=\frac{6-2 \sqrt{5}-5+\sqrt{5}}{5-\sqrt{5}}$

Del apéndice B Sabemos que:

$\cos \left(\theta_{U P}\right)=\sqrt{\frac{5+2 \sqrt{5}}{15}}$

$\operatorname{sen}\left(\theta_{U P}\right)=\sqrt{\frac{10-2 \sqrt{5}}{15}}$

$Y$ de la ecuagsióñ 75 )

$\theta_{D}=2 \cos ^{-1}\left[\frac{5}{\operatorname{sen}\left(\frac{\pi}{5}\right)}\right]-\theta_{U P}$

$\cos (\Psi)=\frac{1-\sqrt{5}}{5-\sqrt{5}} \times \frac{5+\sqrt{5}}{5+\sqrt{5}}$

Dónde:

$\Psi=2 \cos ^{-1}\left[\frac{\cos \left(\frac{2 \pi}{5}\right)}{\operatorname{sen}\left(\frac{\pi}{5}\right)}\right]$

$\cos (\Psi)=\frac{\not \not+\sqrt{5}-5 \sqrt{5}-\not \not b}{25-5}=\frac{-\not \sqrt{5}}{20}$

$\cos (\Psi)=-\frac{\sqrt{5}}{5}$

$\operatorname{sen}(\Psi)=\sqrt{1-\cos ^{2}(\Psi)}$

$\operatorname{sen}(\Psi)=\sqrt{1-\left(-\frac{\sqrt{5}}{5}\right)^{2}}$

$\operatorname{sen}(\Psi)=\sqrt{1-\frac{5}{25}}=\sqrt{\frac{20}{25}}=\frac{\sqrt{4}}{\sqrt{5}} \times \frac{\sqrt{5}}{\sqrt{5}}$

$\cos \left(\frac{\Psi}{2}\right)=\frac{\cos \left(\frac{2 \pi}{5}\right)}{\operatorname{sen}\left(\frac{\pi}{5}\right)}$

$\operatorname{sen}(\Psi)=\frac{2 \sqrt{5}}{5}$ 
Reemplazando (C1), (C2), (C4) y (C5) en (C3)

$$
\begin{aligned}
& \cos \left(\theta_{D}\right)=\left(-\frac{\sqrt{5}}{5}\right)\left(\sqrt{\frac{5+2 \sqrt{5}}{15}}\right)+\left(\frac{2 \sqrt{5}}{5}\right)\left(\sqrt{\frac{10-2 \sqrt{5}}{15}}\right) \\
& \cos \left(\theta_{D}\right)=\left(-\sqrt{\frac{5}{25} \times \frac{5+2 \sqrt{5}}{15}}\right)+\left(\sqrt{\frac{5 \times 4}{25} \times \frac{10-2 \sqrt{5}}{15}}\right)=\ldots \\
& \left(-\sqrt{\frac{1}{5} \times \frac{5+2 \sqrt{5}}{15}}\right)+\left(\sqrt{\frac{4}{5} \times \frac{10-2 \sqrt{5}}{15}}\right) \\
& \cos \left(\theta_{D}\right)=-\frac{1}{5} \sqrt{\frac{1}{3}(5+2 \sqrt{5})}+\frac{1}{5} \sqrt{\frac{8}{3}(5-\sqrt{5})}=-\frac{\sqrt{3}}{15} \sqrt{5+2 \sqrt{5}}+\frac{\sqrt{3}}{15} \sqrt{8(5-\sqrt{5})} \\
& \cos \left(\theta_{D}\right)=\frac{\sqrt{3}}{15}[-\sqrt{5+2 \sqrt{5}}+\sqrt{8(5-\sqrt{5})}]=\frac{\sqrt{3}}{15}[\sqrt{8(5-\sqrt{5})}-\sqrt{5+2 \sqrt{5}}] \\
& \cos ^{2}\left(\theta_{D}\right)=\frac{3}{225}[40-8 \sqrt{5}+5+2 \sqrt{5}-2 \sqrt{8(5-\sqrt{5})(5+2 \sqrt{5})}] \\
& \cos ^{2}\left(\theta_{D}\right)=\frac{3}{225}[45-6 \sqrt{5}-4 \sqrt{2} \sqrt{(5-\sqrt{5})(5+2 \sqrt{5})}] \\
& \cos ^{2}\left(\theta_{D}\right)=\frac{3}{225}[45-6 \sqrt{5}-4 \sqrt{2} \sqrt{25+10 \sqrt{5}-5 \sqrt{5}-10}] \\
& \cos ^{2}\left(\theta_{D}\right)=\frac{3}{225}[45-6 \sqrt{5}-4 \sqrt{2} \sqrt{15+5 \sqrt{5}}]=\frac{3}{225}[45-6 \sqrt{5}-4 \sqrt{10} \sqrt{3+\sqrt{5}}] \\
& \cos ^{2}\left(\theta_{D}\right)=\frac{3}{225}[45-6 \sqrt{5}-4 \sqrt{5} \sqrt{6+2 \sqrt{5}}]=\frac{1}{75}[45-6 \sqrt{5}-4 \sqrt{5}(\sqrt{5}+1)] \\
& \cos ^{2}\left(\theta_{D}\right)=\frac{1}{75}[45-6 \sqrt{5}-20-4 \sqrt{5}]=\frac{1}{75}[25-10 \sqrt{5}]=\frac{5}{75}[5-2 \sqrt{5}] \\
& \cos ^{2}\left(\theta_{D}\right)=\frac{5-2 \sqrt{5}}{15} \\
& \cos \left(\theta_{D}\right)=\sqrt{\frac{5-2 \sqrt{5}}{15}} \\
& \operatorname{sen}^{2}\left(\theta_{D}\right)=1-\frac{5-2 \sqrt{5}}{15}=\frac{15-5+2 \sqrt{5}}{15} \\
& \operatorname{sen}^{2}\left(\theta_{D}\right)=\frac{10+2 \sqrt{5}}{15} \\
& \operatorname{sen}\left(\theta_{D}\right)=\sqrt{\frac{10+2 \sqrt{5}}{15}} \\
& \tan ^{2}\left(\theta_{D}\right)=\frac{2(5+\sqrt{5})}{15} \times \frac{15}{5-2 \sqrt{5}}=\frac{2(5+\sqrt{5})}{5-2 \sqrt{5}} \times \frac{5+2 \sqrt{5}}{5+2 \sqrt{5}} \\
& \tan ^{2}\left(\theta_{D}\right)=\frac{2(25+10+10 \sqrt{5}+5 \sqrt{5})}{25-20}=\frac{2(35+15 \sqrt{5})}{5}=2(7+3 \sqrt{5}) \\
& \tan ^{2}\left(\theta_{D}\right)=14+6 \sqrt{5}=9+6 \sqrt{5}+5=(3+\sqrt{5})^{2} \\
& \tan \left(\theta_{D}\right)=3+\sqrt{5}
\end{aligned}
$$




\section{APÉNDICE D}

$\mathbf{V}_{01}=\mathbf{r}\left[\hat{\mathbf{u}}_{x} \operatorname{sen}\left(\theta_{U P}\right)+\hat{\mathbf{u}}_{z} \cos \left(\theta_{U P}\right)\right]$

$\mathbf{V}_{02}=\mathbf{r}\left[\hat{\mathbf{u}}_{x} \operatorname{sen}\left(\theta_{U P}\right) \cos \left(\frac{2 \pi}{5}\right)+\hat{\mathbf{u}}_{y} \operatorname{sen}\left(\theta_{U P}\right) \operatorname{sen}\left(\frac{2 \pi}{5}\right)+\hat{\mathbf{u}}_{z} \cos \left(\theta_{U P}\right)\right]$

$\mathbf{V}_{03}=\mathbf{r}\left[-\hat{\mathbf{u}}_{x} \operatorname{sen}\left(\theta_{U P}\right) \cos \left(\frac{\pi}{5}\right)+\hat{\mathbf{u}}_{y} \operatorname{sen}\left(\theta_{U P}\right) \operatorname{sen}\left(\frac{\pi}{5}\right)+\hat{\mathbf{u}}_{z} \cos \left(\theta_{U P}\right)\right]$

$\mathbf{V}_{04}=\mathbf{r}\left[-\hat{\mathbf{u}}_{x} \operatorname{sen}\left(\theta_{U P}\right) \cos \left(\frac{\pi}{5}\right)-\hat{\mathbf{u}}_{y} \operatorname{sen}\left(\theta_{U P}\right) \operatorname{sen}\left(\frac{\pi}{5}\right)+\hat{\mathbf{u}}_{z} \cos \left(\theta_{U P}\right)\right]$

$\mathbf{V}_{05}=\mathbf{r}\left[\hat{\mathbf{u}}_{x} \operatorname{sen}\left(\theta_{U P}\right) \cos \left(\frac{2 \pi}{5}\right)-\hat{\mathbf{u}}_{y} \operatorname{sen}\left(\theta_{U P}\right) \operatorname{sen}\left(\frac{2 \pi}{5}\right)+\hat{\mathbf{u}}_{z} \cos \left(\theta_{U P}\right)\right]$

$\mathbf{C}_{1}=\frac{1}{5}\left(\mathbf{V}_{01}+\mathbf{V}_{02}+\mathbf{V}_{03}+\mathbf{V}_{04}+\mathbf{V}_{05}\right)$

$\mathbf{C}_{1}=\frac{\mathbf{r}}{5}\left\{\hat{\mathbf{u}}_{x} \operatorname{sen}\left(\theta_{U P}\right)\left[1+2 \cos \left(\frac{2 \pi}{5}\right)-2 \cos \left(\frac{\pi}{5}\right)\right]+\hat{\mathbf{u}}_{z} 5 \cos \left(\theta_{U P}\right)\right\}$

$\mathbf{C}_{1}=\frac{\mathbf{r}}{5}\left\{\hat{\mathbf{u}}_{x} \operatorname{sen}\left(\theta_{U P}\right)\left[1+\not{Z}\left(\frac{\sqrt{5}-1}{\not A}\right)-\not 2\left(\frac{\sqrt{5}+1}{\not A}\right)\right]+\hat{\mathbf{u}}_{z} 5 \cos \left(\theta_{U P}\right)\right\}$

$\mathbf{C}_{1}=\frac{\mathbf{r}}{5}\left\{\hat{\mathbf{u}}_{x} \operatorname{sen}\left(\theta_{U P}\right)\left[\frac{1}{2}(\not 2+\not \sqrt{5} \not \mathcal{A} \not{5} \not 1)\right]+\hat{\mathbf{u}}_{z} 5 \cos \left(\theta_{U P}\right)\right\}$

$\mathbf{C}_{1}=\frac{\mathbf{r}}{\not \not}\left[\hat{\mathbf{u}}_{z} \not \partial \cos \left(\theta_{U P}\right)\right]$

$\left|\mathbf{C}_{1}\right|=\mathbf{r}\left[\cos \left(\theta_{U P}\right)\right]$

$\hat{\mathbf{C}}_{1}=\frac{\boldsymbol{r}\left[\hat{\mathbf{u}}_{z} \cos \left(\theta_{U P}\right)\right]}{\boldsymbol{r}\left[\cos \left(\theta_{U P}\right)\right]}$

$\cos \Theta_{1}=\frac{\cos \left(\theta_{U P}\right)}{\cos \left(\theta_{U P}\right)}=1$

$\Im_{1}=0^{\circ} \quad ; \pi-\Theta_{1}=180^{\circ}$

$\mathbf{V}_{01}=\mathbf{r}\left[\hat{\mathbf{u}}_{x} \operatorname{sen}\left(\theta_{U P}\right)+\hat{\mathbf{u}}_{z} \cos \left(\theta_{U P}\right)\right]$

$\mathbf{V}_{02}=\mathbf{r}\left[\hat{\mathbf{u}}_{x} \operatorname{sen}\left(\theta_{U P}\right) \cos \left(\frac{2 \pi}{5}\right)+\hat{\mathbf{u}}_{y} \operatorname{sen}\left(\theta_{U P}\right) \operatorname{sen}\left(\frac{2 \pi}{5}\right)+\hat{\mathbf{u}}_{z} \cos \left(\theta_{U P}\right)\right](\mathrm{D} 16)$

$\mathbf{V}_{06}=\mathbf{r}\left[\hat{\mathbf{u}}_{x} \operatorname{sen}\left(\theta_{D}\right)+\hat{\mathbf{u}}_{z} \cos \left(\theta_{D}\right)\right]$

$\mathbf{V}_{07}=\mathbf{r}\left[\hat{\mathbf{u}}_{x} \operatorname{sen}\left(\theta_{D}\right) \cos \left(\frac{2 \pi}{5}\right)+\hat{\mathbf{u}}_{y} \operatorname{sen}\left(\theta_{D}\right) \operatorname{sen}\left(\frac{2 \pi}{5}\right)+\hat{\mathbf{u}}_{z} \cos \left(\theta_{D}\right)\right]$

$\mathbf{V}_{11}=\mathbf{r}\left[\begin{array}{l}\hat{\mathbf{u}}_{x} \operatorname{sen}\left(\pi-\theta_{D}\right) \cos \left(\frac{\pi}{5}\right)+\hat{\mathbf{u}}_{y} \operatorname{sen}\left(\pi-\theta_{D}\right) \operatorname{sen}\left(\frac{\pi}{5}\right)+ \\ \hat{\mathbf{u}}_{z} \cos \left(\pi-\theta_{D}\right)\end{array}\right]$

$\mathrm{C}_{2}=\frac{1}{5}\left(\mathbf{V}_{01}+\mathbf{V}_{02}+\mathbf{V}_{06}+\mathbf{V}_{07}+\mathbf{V}_{11}\right)$

$\operatorname{sen}\left(\pi-\theta_{D}\right)=\operatorname{sen}(\pi) \cos \left(\theta_{D}\right)-\cos (\pi) \operatorname{sen}\left(\theta_{D}\right)$

$\operatorname{sen}\left(\pi-\theta_{D}\right)=\operatorname{sen}\left(\theta_{D}\right)$ 


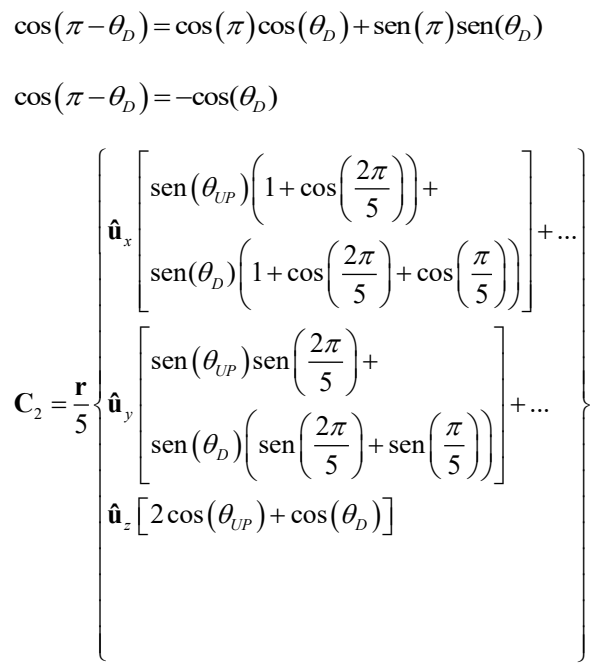

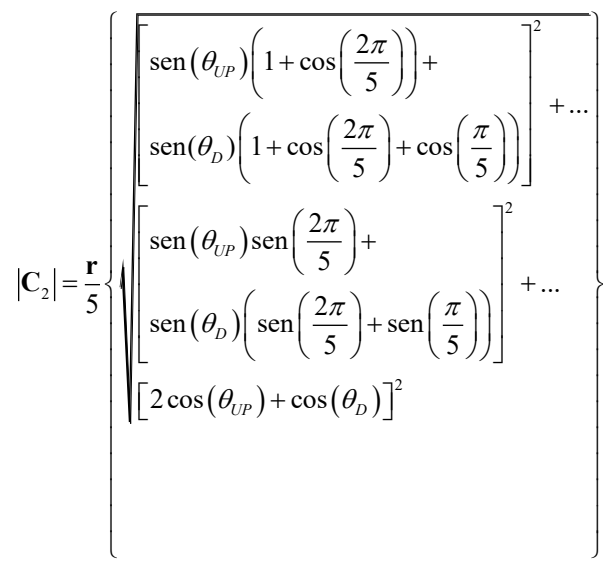

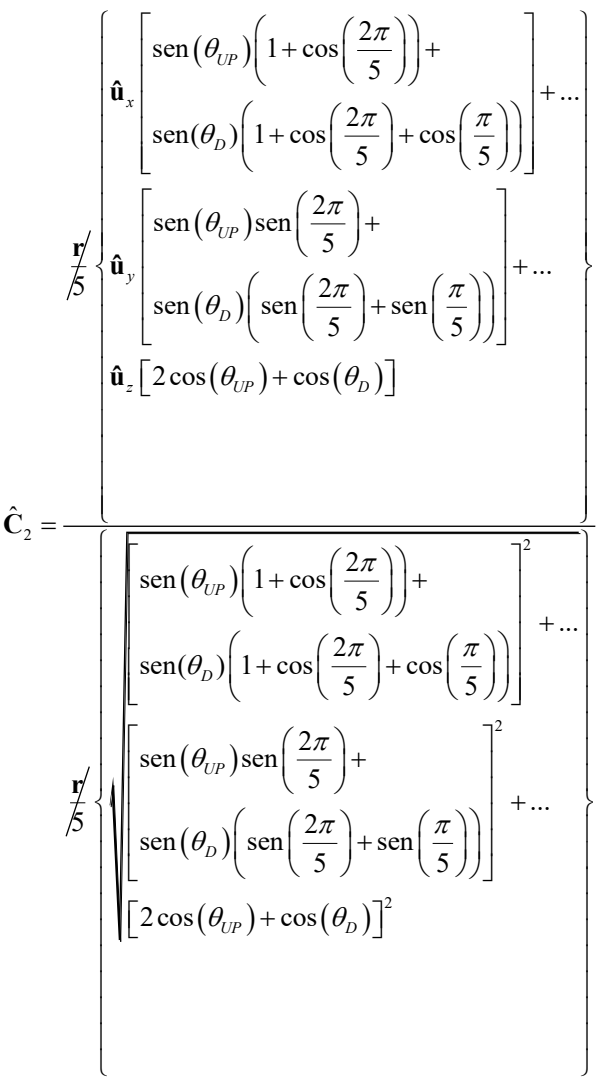




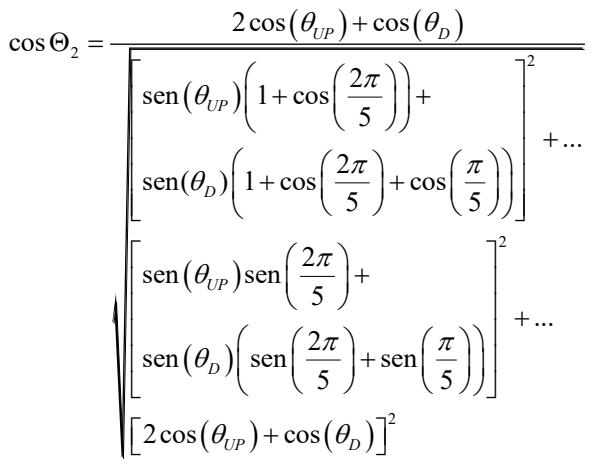

Reemplazando (C1), (C2), (C7), (C9) y Apéndice A en (D28)

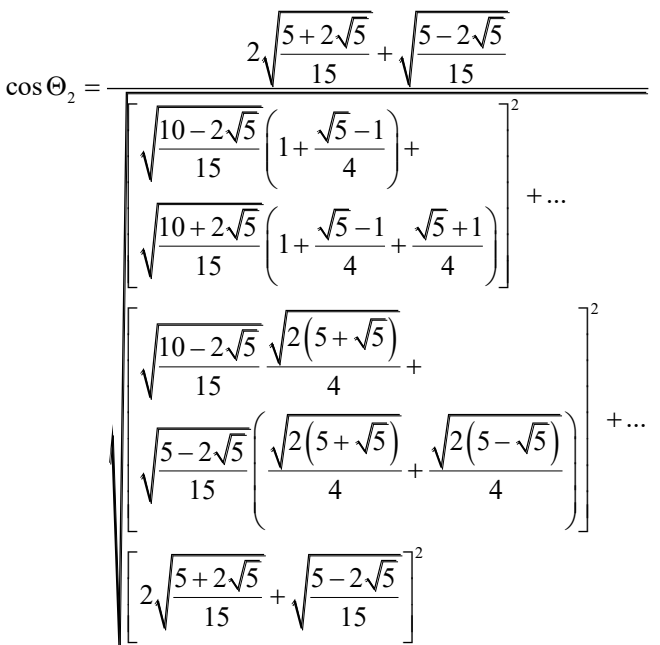

$\Theta_{2}=63.4349^{\circ}$

$\pi-\Theta_{2}=116.5651^{\circ}$

\section{APÉNDICE E}

Identidades trigonométricas:

$$
\begin{aligned}
& \operatorname{sen}^{2}(\alpha)=\frac{1-\cos (2 \alpha)}{2} \\
& \operatorname{sen}(2 \alpha)=2 \operatorname{sen}(\alpha) \cos (\alpha)
\end{aligned}
$$

Fórmulas de diferencia de senos y diferencia de cosenos

$$
\begin{aligned}
& \operatorname{sen}(\alpha+\beta)=\operatorname{sen} \alpha \cos \beta+\cos \alpha \operatorname{sen} \beta \\
& \operatorname{sen}(\alpha-\beta)=\operatorname{sen} \alpha \cos \beta-\cos \alpha \operatorname{sen} \beta \\
& \cos (\alpha+\beta)=\cos \alpha \cos \beta-\operatorname{sen} \alpha \operatorname{sen} \beta \\
& \cos (\alpha-\beta)=\cos \alpha \cos \beta+\operatorname{sen} \alpha \operatorname{sen} \beta
\end{aligned}
$$

Combinación de fórmulas trigonométricas E3 y E4:

$$
\begin{aligned}
& \operatorname{sen}(\alpha+\beta)-\operatorname{sen}(\alpha-\beta)=\operatorname{sen} \alpha \cos \beta+\cos \alpha \operatorname{sen} \beta-(\operatorname{sen} \alpha \cos \beta-\cos \alpha \operatorname{sen} \beta) \\
& \operatorname{sen}(\alpha+\beta)-\operatorname{sen}(\alpha-\beta)=2 \cos \alpha \operatorname{sen} \beta
\end{aligned}
$$

Combinación de fórmulas trigonométricas E5 y E6 nos rinde: 
$\cos (\alpha+\beta)-\cos (\alpha-\beta)=\cos \alpha \cos \beta-\operatorname{sen} \alpha \operatorname{sen} \beta-(\cos \alpha \cos \beta+\operatorname{sen} \alpha \operatorname{sen} \beta)$

$\cos (\alpha+\beta)-\cos (\alpha-\beta)=-2 \operatorname{sen} \alpha \operatorname{sen} \beta$

Producto escalar de vectores (Fórmula para obtener el

ángulo entre dos vectores:

)

Figura 15

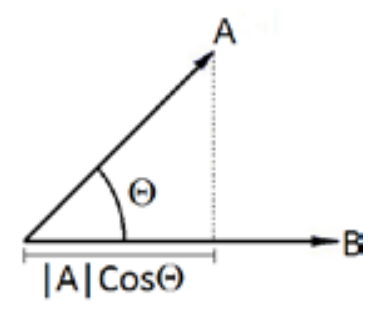

vectores
Producto escalar de

A.B $=|A| \cdot|B| \cos \Theta$

$\frac{\mathrm{A} \cdot \mathrm{B}}{|\mathrm{A}| \cdot|\mathrm{B}|}=\cos \Theta$

Fórmula del ángulo interno de un polígono:

$\Theta=\frac{(n-2) 180}{n}$

Donde el número de lados " $n$ " igual a 5 para un pentágono, el ángulo “ $\Theta$ ” interno es:

$\Theta=\frac{3 \pi}{5}=108^{\circ}$

Coseno de $\Theta$ es:

) $\cos \left(\frac{3 \pi}{5}\right)=\cos \left(\frac{5 \pi}{2}-\frac{2 \pi}{5}\right)=\cos (\pi) \cos \left(\frac{2 \pi}{5}\right)+\operatorname{sen}(\pi) \operatorname{sen}\left(\frac{2 \pi}{5}\right)$

$\cos \left(\frac{3 \pi}{5}\right)=-\cos \left(\frac{2 \pi}{5}\right) \therefore$

Fórmula del producto escalar de vectores:

$\hat{\mathbf{u}}_{\mathrm{x}} \cdot \hat{\mathbf{u}}_{\mathrm{x}}=\hat{\mathbf{u}}_{\mathrm{y}} \cdot \hat{\mathbf{u}}_{\mathrm{y}}=\hat{\mathbf{u}}_{\mathrm{z}} \cdot \hat{\mathbf{u}}_{\mathrm{z}}=1 \cdot 1 \cos 0=1$

$\hat{\mathbf{u}}_{x} \cdot \hat{\mathbf{u}}_{y}=\hat{\mathbf{u}}_{x} \cdot \hat{\mathbf{u}}_{z}=\hat{\mathbf{u}}_{y} \cdot \hat{\mathbf{u}}_{z}=1 \cdot 1 \cos \frac{\pi}{2}=0$

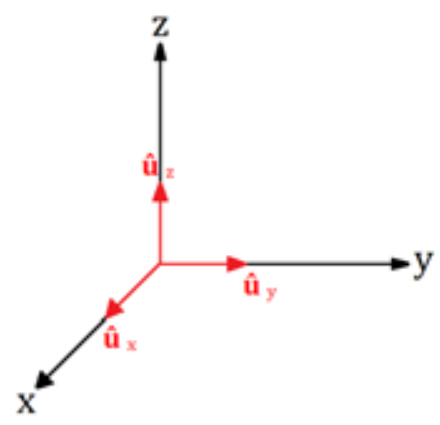

Figura 16. Vectores unitarios $\hat{\mathbf{u}}_{\mathrm{x}}, \hat{\mathbf{u}}_{\mathrm{y}}, \hat{\mathbf{u}}_{\mathrm{z}}$

Identidades trigonométricas:

$\cos (2 \alpha)=1-2 \sin ^{2}(\alpha)$ 


\section{APÉNDICE F}

Debido a la necesidad de efectuar cálculos con las funciones trigonométricas de $\frac{\pi}{5}, \frac{2 \pi}{5}, \frac{3 \pi}{5}, \frac{4 \pi}{5}, \frac{6 \pi}{5}, \frac{7 \pi}{5}, \frac{8 \pi}{5}$ y $\frac{9 \pi}{5}$ en la configuración (2) para determinar el valor del ángulo $\theta_{D} \mathrm{y}$ $\theta_{U P}$, En el apéndice A se demuestra, a partir de primeros principios, que:

$$
\begin{aligned}
& \cos \left(\frac{3 \pi}{5}\right)=-\cos \left(\frac{2 \pi}{5}\right) \\
& \cos \left(\frac{4 \pi}{5}\right)=-\cos \left(\frac{\pi}{5}\right) \\
& \cos \left(\frac{6 \pi}{5}\right)=-\cos \left(\frac{\pi}{5}\right) \\
& \cos \left(\frac{7 \pi}{5}\right)=-\cos \left(\frac{2 \pi}{5}\right) \\
& \cos \left(\frac{8 \pi}{5}\right)=\cos \left(\frac{2 \pi}{5}\right) \\
& \cos \left(\frac{9 \pi}{5}\right)=\cos \left(\frac{\pi}{5}\right) \\
& \operatorname{sen}\left(\frac{3 \pi}{5}\right)=\operatorname{sen}\left(\frac{2 \pi}{5}\right) \\
& \operatorname{sen}\left(\frac{4 \pi}{5}\right)=\operatorname{sen}\left(\frac{\pi}{5}\right) \\
& \operatorname{sen}\left(\frac{6 \pi}{5}\right)=-\operatorname{sen}\left(\frac{\pi}{5}\right) \\
& \operatorname{sen}\left(\frac{7 \pi}{5}\right)=-\operatorname{sen}\left(\frac{2 \pi}{5}\right) \\
& \operatorname{sen}\left(\frac{8 \pi}{5}\right)=-\operatorname{sen}\left(\frac{2 \pi}{5}\right) \\
& \operatorname{sen}\left(\frac{9 \pi}{5}\right)=-\operatorname{sen}\left(\frac{\pi}{5}\right)
\end{aligned}
$$

A partir de la configuración (2) se desprenden las ecuaciones correspondientes de todos los vértices que son:

$$
\begin{gathered}
\theta_{1}=\theta_{2}=\theta_{3}=\theta_{4}=\theta_{5}=\theta_{\mathrm{UP}} \\
\varphi_{1}=0, \varphi_{2}=2 \pi / 5, \varphi_{3}=4 \pi / 5, \varphi_{4}=6 \pi / 5, \varphi_{5}=8 \pi / 5 \\
\mathbf{V}_{01}=\mathrm{r}\left[\hat{\mathbf{u}}_{x} \operatorname{sen}\left(\theta_{U P}\right) \cos (0)+\hat{\mathbf{u}}_{y} \operatorname{sen}\left(\theta_{U P}\right) \operatorname{sen}(0)+\hat{\mathbf{u}}_{z} \cos \left(\theta_{U P}\right)\right] \\
\mathbf{V}_{02}=\mathrm{r}\left[\hat{\mathbf{u}}_{x} \operatorname{sen}\left(\theta_{U P}\right) \cos \left(\frac{2 \pi}{5}\right)+\hat{\mathbf{u}}_{y} \operatorname{sen}\left(\theta_{U P}\right) \operatorname{sen}\left(\frac{2 \pi}{5}\right)+\hat{\mathbf{u}}_{z} \cos \left(\theta_{U P}\right)\right] \\
\mathbf{V}_{03}=\mathrm{r}\left[\hat{\mathbf{u}}_{x} \operatorname{sen}\left(\theta_{U P}\right) \cos \left(\frac{4 \pi}{5}\right)+\hat{\mathbf{u}}_{y} \operatorname{sen}\left(\theta_{U P}\right) \operatorname{sen}\left(\frac{4 \pi}{5}\right)+\hat{\mathbf{u}}_{z} \cos \left(\theta_{U P}\right)\right]
\end{gathered}
$$




$$
\begin{aligned}
& \mathbf{V}_{04}=\mathrm{r}\left[\hat{\mathbf{u}}_{x} \operatorname{sen}\left(\theta_{U P}\right) \cos \left(\frac{6 \pi}{5}\right)+\hat{\mathbf{u}}_{y} \operatorname{sen}\left(\theta_{U P}\right) \operatorname{sen}\left(\frac{6 \pi}{5}\right)+\hat{\mathbf{u}}_{z} \cos \left(\theta_{U P}\right)\right] \\
& \mathbf{V}_{05}=\mathrm{r}\left[\hat{\mathbf{u}}_{x} \operatorname{sen}\left(\theta_{U P}\right) \cos \left(\frac{8 \pi}{5}\right)+\hat{\mathbf{u}}_{y} \operatorname{sen}\left(\theta_{U P}\right) \operatorname{sen}\left(\frac{8 \pi}{5}\right)+\hat{\mathbf{u}}_{z} \cos \left(\theta_{U P}\right)\right]
\end{aligned}
$$

$$
\theta_{6}=\theta_{7}=\theta_{8}=\theta_{9}=\theta_{10}=\theta_{\mathrm{D}} ;
$$

$\varphi_{6}=0, \varphi_{7}=2 \pi / 5, \varphi_{8}=4 \pi / 5, \varphi_{9}=6 \pi / 5, \varphi_{10}=8 \pi / 5$;

$$
\begin{aligned}
& \mathbf{V}_{06}=\mathrm{r}\left[\hat{\mathbf{u}}_{x} \operatorname{sen}\left(\theta_{D}\right) \cos (0)+\hat{\mathbf{u}}_{y} \operatorname{sen}\left(\theta_{D}\right) \operatorname{sen}(0)+\hat{\mathbf{u}}_{z} \cos \left(\theta_{D}\right)\right] \\
& \mathbf{V}_{07}=\mathrm{r}\left[\hat{\mathbf{u}}_{x} \operatorname{sen}\left(\theta_{D}\right) \cos \left(\frac{2 \pi}{5}\right)+\hat{\mathbf{u}}_{y} \operatorname{sen}\left(\theta_{D}\right) \operatorname{sen}\left(\frac{2 \pi}{5}\right)+\hat{\mathbf{u}}_{z} \cos \left(\theta_{D}\right)\right] \\
& \mathbf{V}_{08}=\mathrm{r}\left[\hat{\mathbf{u}}_{x} \operatorname{sen}\left(\theta_{D}\right) \cos \left(\frac{4 \pi}{5}\right)+\hat{\mathbf{u}}_{y} \operatorname{sen}\left(\theta_{D}\right) \operatorname{sen}\left(\frac{4 \pi}{5}\right)+\hat{\mathbf{u}}_{z} \cos \left(\theta_{D}\right)\right] \\
& \mathbf{V}_{09}=\mathrm{r}\left[\hat{\mathbf{u}}_{x} \operatorname{sen}\left(\theta_{D}\right) \cos \left(\frac{6 \pi}{5}\right)+\hat{\mathbf{u}}_{y} \operatorname{sen}\left(\theta_{D}\right) \operatorname{sen}\left(\frac{6 \pi}{5}\right)+\hat{\mathbf{u}}_{z} \cos \left(\theta_{D}\right)\right] \\
& \mathbf{V}_{10}=\mathrm{r}\left[\hat{\mathbf{u}}_{x} \operatorname{sen}\left(\theta_{D}\right) \cos \left(\frac{8 \pi}{5}\right)+\hat{\mathbf{u}}_{y} \operatorname{sen}\left(\theta_{D}\right) \operatorname{sen}\left(\frac{8 \pi}{5}\right)+\hat{\mathbf{u}}_{z} \cos \left(\theta_{D}\right)\right]
\end{aligned}
$$

$$
\begin{gathered}
\theta_{11}=\theta_{12}=\theta_{13}=\theta_{14}=\theta_{15} \equiv \pi-\theta_{\mathrm{D}} \\
\varphi_{11}=\pi / 5, \varphi_{12}=3 \pi / 5, \varphi_{13}=\pi, \varphi_{14}=7 \pi / 5, \varphi_{15}=9 \pi / 5
\end{gathered}
$$

$$
\mathbf{V}_{11}=\mathrm{r}\left[\begin{array}{l}
\hat{\mathbf{u}}_{x} \operatorname{sen}\left(\pi-\theta_{D}\right) \cos \left(\frac{\pi}{5}\right)+\hat{\mathbf{u}}_{y} \operatorname{sen}\left(\pi-\theta_{D}\right) \operatorname{sen}\left(\frac{\pi}{5}\right)+ \\
\hat{\mathbf{u}}_{z} \cos \left(\pi-\theta_{D}\right)
\end{array}\right]
$$

$$
\mathbf{V}_{12}=\mathrm{r}\left[\begin{array}{l}
\hat{\mathbf{u}}_{x} \operatorname{sen}\left(\pi-\theta_{D}\right) \cos \left(\frac{3 \pi}{5}\right)+\hat{\mathbf{u}}_{y} \operatorname{sen}\left(\pi-\theta_{D}\right) \operatorname{sen}\left(\frac{3 \pi}{5}\right)+ \\
\hat{\mathbf{u}}_{z} \cos \left(\pi-\theta_{D}\right)
\end{array}\right]
$$

$$
\mathbf{V}_{13}=\mathrm{r}\left[\begin{array}{l}
\hat{\mathbf{u}}_{x} \operatorname{sen}\left(\pi-\theta_{D}\right) \cos (\pi)+\hat{\mathbf{u}}_{y} \operatorname{sen}\left(\pi-\theta_{D}\right) \operatorname{sen}(\pi)+ \\
\hat{\mathbf{u}}_{z} \cos \left(\pi-\theta_{D}\right)
\end{array}\right]
$$

$\mathbf{V}_{14}=\mathrm{r}\left[\begin{array}{l}\hat{\mathbf{u}}_{x} \operatorname{sen}\left(\pi-\theta_{D}\right) \cos \left(\frac{7 \pi}{5}\right)+\hat{\mathbf{u}}_{y} \operatorname{sen}\left(\pi-\theta_{D}\right) \operatorname{sen}\left(\frac{7 \pi}{5}\right)+ \\ \hat{\mathbf{u}}_{z} \cos \left(\pi-\theta_{D}\right)\end{array}\right]$

$$
\mathbf{V}_{15}=\mathrm{r}\left[\begin{array}{l}
\hat{\mathbf{u}}_{x} \operatorname{sen}\left(\pi-\theta_{D}\right) \cos \left(\frac{9 \pi}{5}\right)+\hat{\mathbf{u}}_{y} \operatorname{sen}\left(\pi-\theta_{D}\right) \operatorname{sen}\left(\frac{9 \pi}{5}\right)+ \\
\hat{\mathbf{u}}_{z} \cos \left(\pi-\theta_{D}\right)
\end{array}\right]
$$

$$
\begin{gathered}
\theta_{16}=\theta_{17}=\theta_{18}=\theta_{19}=\theta_{20} \equiv \pi-\theta_{\mathrm{UP}} \\
\varphi_{16}=\pi / 5, \varphi_{17}=3 \pi / 5, \varphi_{18}=\pi, \varphi_{19}=7 \pi / 5, \varphi_{20} \quad 9 \pi / 5 . \\
\mathbf{V}_{16}=\mathrm{r}\left[\begin{array}{l}
\left.\hat{\mathbf{u}}_{x} \operatorname{sen}\left(\pi-\theta_{U P}\right) \cos \left(\frac{\pi}{5}\right)+\hat{\mathbf{u}}_{y} \operatorname{sen}\left(\pi-\theta_{U P}\right) \operatorname{sen}\left(\frac{\pi}{5}\right)+\right] \\
\hat{\mathbf{u}}_{z} \cos \left(\pi-\theta_{U P}\right)
\end{array}\right] \\
\mathbf{V}_{17}=\mathrm{r}\left[\begin{array}{l}
\left.\hat{\mathbf{u}}_{x} \operatorname{sen}\left(\pi-\theta_{U P}\right) \cos \left(\frac{3 \pi}{5}\right)+\hat{\mathbf{u}}_{y} \operatorname{sen}\left(\pi-\theta_{U P}\right) \operatorname{sen}\left(\frac{3 \pi}{5}\right)+\right] \\
\hat{\mathbf{u}}_{z} \cos \left(\pi-\theta_{U P}\right)
\end{array}\right]
\end{gathered}
$$




$$
\begin{aligned}
& \mathbf{V}_{18}=\mathrm{r}\left[\begin{array}{l}
\hat{\mathbf{u}}_{x} \operatorname{sen}\left(\pi-\theta_{U P}\right) \cos (\pi)+\hat{\mathbf{u}}_{y} \operatorname{sen}\left(\pi-\theta_{U P}\right) \operatorname{sen}(\pi)+ \\
\hat{\mathbf{u}}_{z} \cos \left(\pi-\theta_{U P}\right)
\end{array}\right] \\
& \mathbf{V}_{19}=\mathrm{r}\left[\begin{array}{l}
\hat{\mathbf{u}}_{x} \operatorname{sen}\left(\pi-\theta_{U P}\right) \cos \left(\frac{7 \pi}{5}\right)+\hat{\mathbf{u}}_{y} \operatorname{sen}\left(\pi-\theta_{U P}\right) \operatorname{sen}\left(\frac{7 \pi}{5}\right)+ \\
\hat{\mathbf{u}}_{z} \cos \left(\pi-\theta_{U P}\right)
\end{array}\right] \\
& \mathbf{V}_{20}=\mathrm{r}\left[\begin{array}{l}
\hat{\mathbf{u}}_{x} \operatorname{sen}\left(\pi-\theta_{U P}\right) \cos \left(\frac{9 \pi}{5}\right)+\hat{\mathbf{u}}_{y} \operatorname{sen}\left(\pi-\theta_{U P}\right) \operatorname{sen}\left(\frac{9 \pi}{5}\right)+ \\
\hat{\mathbf{u}}_{z} \cos \left(\pi-\theta_{U P}\right)
\end{array}\right]
\end{aligned}
$$

A partir de las ecuaciones (F1) a (F20) y de las ecuaciones (A5) a (A16) las coordenadas cartesianas de los 20 vértices son:

$$
\begin{aligned}
& \mathbf{V}_{01}=\mathrm{r}\left[\operatorname{sen}\left(\theta_{U P}\right), 0, \cos \left(\theta_{U P}\right)\right] \\
& \mathbf{V}_{02}=\mathrm{r}\left[\operatorname{sen}\left(\theta_{U P}\right) \cos \left(\frac{2 \pi}{5}\right), \operatorname{sen}\left(\theta_{U P}\right) \operatorname{sen}\left(\frac{2 \pi}{5}\right), \cos \left(\theta_{U P}\right)\right] \\
& \mathbf{V}_{03}=\mathrm{r}\left[-\operatorname{sen}\left(\theta_{U P}\right) \cos \left(\frac{\pi}{5}\right), \operatorname{sen}\left(\theta_{U P}\right) \operatorname{sen}\left(\frac{\pi}{5}\right), \cos \left(\theta_{U P}\right)\right] \\
& \mathbf{V}_{04}=\mathrm{r}\left[-\operatorname{sen}\left(\theta_{U P}\right) \cos \left(\frac{\pi}{5}\right),-\operatorname{sen}\left(\theta_{U P}\right) \operatorname{sen}\left(\frac{\pi}{5}\right), \cos \left(\theta_{U P}\right)\right] \\
& \mathbf{V}_{05}=\mathrm{r}\left[\operatorname{sen}\left(\theta_{U P}\right) \cos \left(\frac{2 \pi}{5}\right),-\operatorname{sen}\left(\theta_{U P}\right) \operatorname{sen}\left(\frac{2 \pi}{5}\right), \cos \left(\theta_{U P}\right)\right] \\
& \mathbf{V}_{06}=\mathrm{r}\left[\operatorname{sen}\left(\theta_{D}\right), 0, \cos \left(\theta_{D}\right)\right] \\
& \mathbf{V}_{07}=\mathrm{r}\left[\operatorname{sen}\left(\theta_{D}\right) \cos \left(\frac{2 \pi}{5}\right), \operatorname{sen}\left(\theta_{D}\right) \operatorname{sen}\left(\frac{2 \pi}{5}\right), \cos \left(\theta_{D}\right)\right] \\
& \mathbf{V}_{15}=\mathrm{r}\left[\operatorname{sen}\left(\pi-\theta_{D}\right) \cos \left(\frac{\pi}{5}\right),-\operatorname{sen}\left(\pi-\theta_{D}\right) \operatorname{sen}\left(\frac{\pi}{5}\right), \cos \left(\pi-\theta_{D}\right)\right] \\
& \mathbf{V}_{08}=\mathrm{r}\left[-\operatorname{sen}\left(\theta_{D}\right) \cos \left(\frac{\pi}{5}\right), \operatorname{sen}\left(\theta_{D}\right) \operatorname{sen}\left(\frac{\pi}{5}\right), \cos \left(\theta_{D}\right)\right] \\
& \mathbf{V}_{13}=\mathrm{r}\left[-\operatorname{sen}\left(\pi-\theta_{D}\right), 0, \cos \left(\pi-\theta_{D}\right)\right] \\
& \mathbf{V}_{11}=\mathrm{r}\left[-\operatorname{sen}\left(\pi-\theta_{D}\right) \cos \left(\frac{2 \pi}{5}\right),-\operatorname{sen}\left(\pi-\theta_{D}\right) \operatorname{sen}\left(\frac{2 \pi}{5}\right), \cos \left(\pi-\theta_{D}\right)\right] \\
& \mathbf{V}_{10}=\mathrm{r}\left[\operatorname{sen}\left(\pi-\theta_{D}\right) \cos \left(\frac{\pi}{5}\right), \operatorname{sen}\left(\pi-\theta_{D}\right) \cos \left(\frac{\pi}{5}\right),-\operatorname{sen}\left(\theta_{D}\right) \operatorname{sen}\left(\frac{\pi}{5}\right), \cos \left(\frac{\pi}{5}\right), \cos \left(\theta_{D}\right)\right]
\end{aligned}
$$




$$
\begin{aligned}
& \mathbf{V}_{16}=\mathrm{r}\left[\operatorname{sen}\left(\pi-\theta_{U P}\right) \cos \left(\frac{\pi}{5}\right), \operatorname{sen}\left(\pi-\theta_{U P}\right) \operatorname{sen}\left(\frac{\pi}{5}\right), \cos \left(\pi-\theta_{U P}\right)\right](\mathrm{F} 36) \\
& \mathbf{V}_{17}=\mathrm{r}\left[-\operatorname{sen}\left(\pi-\theta_{U P}\right) \cos \left(\frac{2 \pi}{5}\right), \operatorname{sen}\left(\pi-\theta_{U P}\right) \operatorname{sen}\left(\frac{2 \pi}{5}\right), \cos \left(\pi-\theta_{U P}\right)\right](\mathrm{F} 37) \\
& \mathbf{V}_{18}=\mathrm{r}\left[-\operatorname{sen}\left(\pi-\theta_{U P}\right), 0, \cos \left(\pi-\theta_{U P}\right)\right] \\
& \mathbf{V}_{19}=\mathrm{r}\left[-\operatorname{sen}\left(\pi-\theta_{U P}\right) \cos \left(\frac{2 \pi}{5}\right),-\operatorname{sen}\left(\pi-\theta_{U P}\right) \operatorname{sen}\left(\frac{2 \pi}{5}\right),\right] \\
& \mathbf{V}_{20}=\mathrm{r}\left[\cos \left(\pi-\theta_{U P}\right)\right. \\
& \left.\operatorname{sen}\left(\pi-\theta_{U P}\right) \cos \left(\frac{\pi}{5}\right),-\operatorname{sen}\left(\pi-\theta_{U P}\right) \operatorname{sen}\left(\frac{\pi}{5}\right), \cos \left(\pi-\theta_{U P}\right)\right]
\end{aligned}
$$

